\title{
A reference point invariant Lamb vector based aerodynamic force breakdown in steady compressible flows
}

\author{
Camille Fournis* and Didier Bailly ${ }^{\dagger}$ \\ ONERA-The French Aerospace Lab, 92190 Meudon, France \\ Renato Tognaccini $\mathrm{i}^{+}$ \\ Università di Napoli Federico II, Napoli, 80125, Italia
}

\begin{abstract}
Some recent developments in the Lamb vector based aerodynamic force breakdown used the concept of vortex force in order to define the lift and to decompose the drag into lift-induced drag and profile drag. However, the Lamb vector formulation is based on moments and the associated force breakdown may depend on the reference point adopted for their computation. Yet, the force acting on an airplane cannot be dependent on this point. Thus, a systematic method based on the far field flow symmetries is here proposed in order to get rid of this dependence. A new formulation is then defined and tested for a two-dimensional transonic flow around an OAT15A airfoil and a three-dimensional flow around the wing of the NASA Common Research Model.
\end{abstract}

\section{Nomenclature}

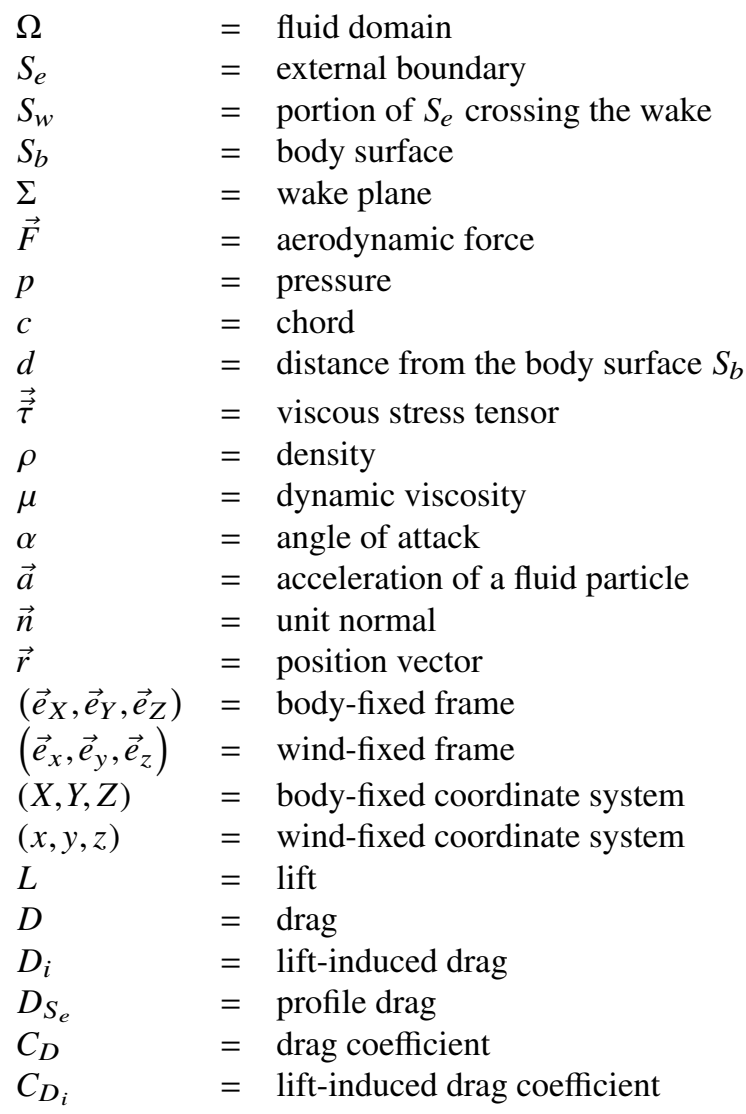

*Ph.D student, Aerodynamics, Aeroelasticity and Acoustics Department, camille.fournis@onera.fr.

${ }^{\dagger}$ Research Engineer, Aerodynamics, Aeroelasticity and Acoustics Department, didier.bailly@onera.fr.

¥Associate Professor, Dipartimento di Ingegneria Industriale, renato.tognaccini@ unina.it, AIAA Senior Member. 


$\begin{array}{ll}C_{D_{v}} & =\text { viscous drag coefficient } \\ C_{D_{w}} & =\text { wave drag coefficient } \\ C_{D_{\rho l}} & =\text { drag coefficient of the vortex force } \\ C_{D_{m_{\rho}}} & =\text { drag coefficient of the compressibility correction } \\ C_{D_{S}} & =\text { profile drag coefficient } \\ \nabla & =\text { gradient operator } \nabla=\frac{\partial}{\partial x} \vec{e}_{x}+\frac{\partial}{\partial y} \vec{e}_{y}+\frac{\partial}{\partial z} \vec{e}_{z} \\ \vec{u} & =\text { velocity vector } \vec{u}=u_{x} \vec{e}_{x}+u_{y} \vec{e}_{y}+u_{z} \vec{e}_{z} \\ \vec{\omega} & =\text { vorticity vector } \vec{\omega}=\nabla \times \vec{u} \\ \vec{l} & =\text { lamb vector } \vec{l}=\vec{\omega} \times \vec{u} \\ n & =\text { dimension of the physical space } n=2,3 \\ u^{2} & =\text { velocity squared } u^{2}=u_{x}^{2}+u_{y}^{2}+u_{z}^{2} \\ \vec{U}_{\infty} & =\text { freestream velocity } \vec{U}_{\infty}=U_{\infty} \vec{e}_{x} \\ a_{\infty} & =\text { freestream speed of sound } \\ M_{\infty} & =\text { freestream Mach number } M_{\infty}=U_{\infty} / a_{\infty} \\ R e & =\text { Reynolds number } R e=\left(\rho_{\infty} U_{\infty} c\right) / \mu_{\infty}\end{array}$

\section{Introduction}

$\Gamma^{H E}$ determination of the total aerodynamic force acting on a body has been of paramount importance since the birth of aviation in the late nineteenth and early twentieth century. An accurate computation of the aerodynamic drag is a major issue when designing an aircraft since it has a significant impact on its global performance, total weight and fuel consumption.

There are two ways of computing the aerodynamic force: the near field and the far field approach. The former consists in integrating the pressure and shear stresses acting on the body skin $S_{b}$ whereas the latter is based on the momentum balance in a control volume of fluid $\Omega$ and its external boundary $S_{e}$ (see Fig 1 ):

$$
\vec{F}=-\int_{S_{b}}(-p \overrightarrow{\overrightarrow{1}}+\overrightarrow{\vec{\tau}}) \cdot \vec{n} \mathrm{~d} S=-\int_{\Omega} \rho \vec{a} \mathrm{~d} v+\int_{S_{e}}(-p \overrightarrow{\overrightarrow{1}}+\overrightarrow{\vec{\tau}}) \cdot \vec{n} \mathrm{~d} S
$$

where $\vec{a}$ is the acceleration of a fluid particle, $\overrightarrow{\vec{\tau}}$ is the viscous stress tensor and $\vec{n}$ is the unit normal to the surface pointing outside the fluid.

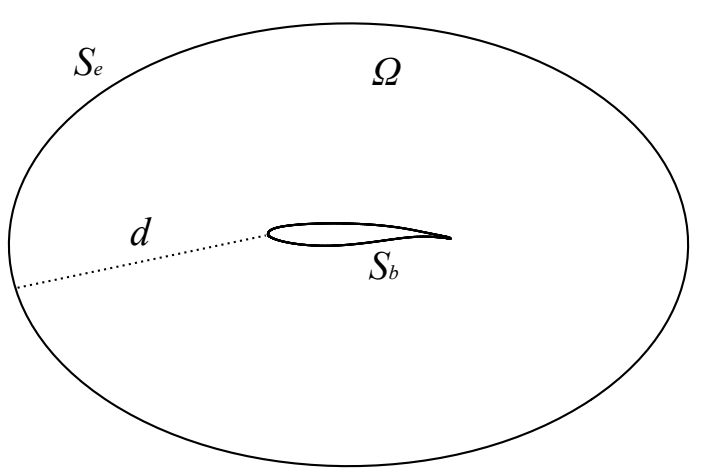

(a) $S_{e}$ defined by the distance from the body

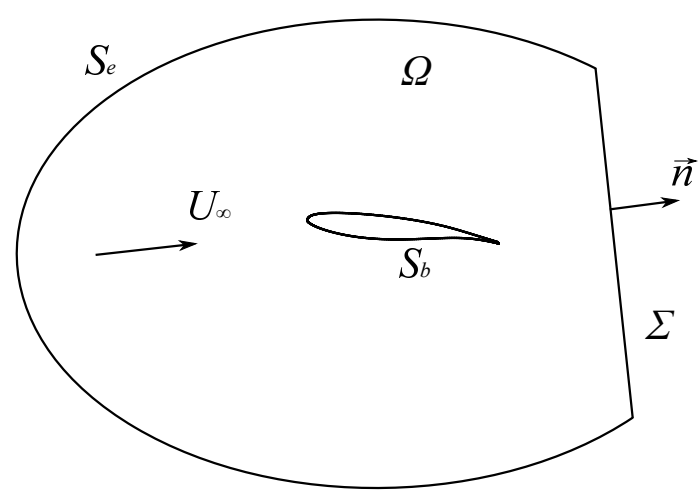

(b) $S_{e}$ defined with a wake plane $\Sigma$

Fig. 1 Fluid domain and its boundaries

Far field methods provide several advantages:

- A decomposition of the aerodynamic drag into several terms related to the physical phenomena occuring in the flow: trailing vortices, viscous wakes and shock waves. Hence the total drag is given by the sum of three contributions, namely the induced drag, the viscous drag and the wave drag: $C_{D}=C_{D_{i}}+C_{D_{v}}+C_{D_{w}}$.

- The identification of the local flow structures where the different drag contributions are generated in the flow field. 
- An estimate of the spurious drag which allows for a more accurate calculation of the total drag when dealing with numerical computations.

The far field decomposition of the aerodynamic drag has been a matter of research in the last two decades with the development of a thermodynamic method [1, 2] based on the entropy production within the boundary layers and across shock waves. This formula generalizes the concept of entropy drag first introduced by Oswatitsch [3] in 1956 and provides a consistent breakdown of the profile drag into viscous and wave drag contributions. The main drawback of this formulation lies in the fact that it does not define explicitly and cannot accurately compute the lift-induced drag since it is found by subtracting the profile drag to the total drag.

The vorticity-based formulations form another family of the far field methods and have been subject to intense research in the very recent years (an exhaustive summary is presented in [4]) since they provide a consistent definition of the lift and the lift-induced drag. The vortex force theory was first introduced by Prandtl [5] when he developed his well-known inviscid lifting-line theory and was discussed by Saffman [6] in the case of an incompressible steady inviscid flow. In their derivations the pressure is replaced by the kinetic energy upon using the Bernoulli equation and the aerodynamic force is expressed with the Lamb vector field $\vec{l}=\vec{\omega} \times \vec{u}$ where $\vec{\omega}=\nabla \times \vec{u}$ is the vorticity. The extension to the relevant case of the body wake convected infinitely downstream also including the steady case was conducted by Wu et al. [7, 8] where the pressure field is eliminated by using the derivative moment equation Eq. 47):

$$
\vec{F}=-\int_{\Omega} \rho \vec{l} \mathrm{~d} v-\frac{1}{n-1} \int_{S_{e}} \vec{r} \times(\vec{n} \times \rho \vec{l}) \mathrm{d} S+\frac{1}{n-1} \int_{S_{e}} \vec{r} \times(\vec{n} \times \nabla \cdot \overrightarrow{\vec{\tau}}) \mathrm{d} S+\int_{S_{e}} \overrightarrow{\vec{\tau}} \cdot \vec{n} \mathrm{~d} S
$$

where $n=2,3$ is the dimension of the space and $\vec{r}$ is the position vector. A compressible version of the above-mentioned formulation was suggested by Wu et al. [7] (p. 621) and by Liu et al. [9] who defined a compressible Lamb vector $\rho \vec{l}-\frac{u^{2}}{2} \nabla \rho$ such that:

$$
\vec{F}=-\int_{\Omega}\left(\rho \vec{l}-\frac{u^{2}}{2} \nabla \rho\right) \mathrm{d} v-\frac{1}{n-1} \int_{S_{e}} \vec{r} \times\left[\vec{n} \times\left(\rho \vec{l}-\frac{u^{2}}{2} \nabla \rho\right)\right] \mathrm{d} S+\frac{1}{n-1} \int_{S_{e}} \vec{r} \times(\vec{n} \times \nabla \cdot \overrightarrow{\vec{\tau}}) \mathrm{d} S+\int_{S_{e}} \overrightarrow{\vec{\tau}} \cdot \vec{n} \mathrm{~d} S
$$

In incompressible flows, the integration can be limited to regions where the vorticity is not zero since only the Lamb vector appears whereas in compressible flows, one loses the compactness of the integrands because of the term $\frac{u^{2}}{2} \nabla \rho$. Hence, Mele and Tognaccini [10] used Eq.(46] in order to express Eq.(3) as follows:

$$
\vec{F}=\vec{F}_{\rho l}+\vec{F}_{m_{\rho}}+\vec{F}_{S_{e}}+\vec{F}_{\tau}
$$

where

$$
\begin{aligned}
\vec{F}_{\rho l} & =-\int_{\Omega} \rho \vec{l} \mathrm{~d} v \\
\vec{F}_{m_{\rho}} & =-\frac{1}{n-1} \int_{\Omega} \vec{r} \times\left(\nabla \rho \times \nabla\left(\frac{u^{2}}{2}\right)\right) \mathrm{d} v \\
\vec{F}_{S e} & =-\frac{1}{n-1} \int_{S_{e}} \vec{r} \times(\vec{n} \times \rho \vec{l}) \mathrm{d} S \\
\vec{F}_{\tau} & =\frac{1}{n-1} \int_{S_{e}} \vec{r} \times(\vec{n} \times \nabla \cdot \overrightarrow{\vec{\tau}}) \mathrm{d} S+\int_{S_{e}} \overrightarrow{\vec{\tau}} \cdot \vec{n} \mathrm{~d} S
\end{aligned}
$$

$\vec{F}_{\rho l}$ is the vortex force which provides the lift $L$ and the lift-induced drag $D_{i}$ in steady incompressible flows [11], $\vec{F}_{m}$ is a compressibility correction term to the vortex force which contributes to $L$ and $D_{i}$ in steady compressible flows [10] and $\vec{F}_{S e}$ provides the profile drag $D_{S_{e}}$ as noticed by several authors [7, 10]. Finally, for a large enough control volume, $S_{e}$ retreats to flow regions where viscous stresses become negligible such that $\vec{F}_{\tau}$ can be dropped [11, 12]. The profile drag consists of a viscous and a wave contribution: the viscous drag is produced in the boundary layers while the wave drag is produced across shock waves. Therefore, distinguishing the two contributions turns out to be tough as noticed by Mele et al. [13] who proposed to define the wave drag as the surface integral $\vec{F}_{S e}$ on a wake plane directly downstream of the shock. The viscous drag was then computed subtracting the wave drag to the profile drag. Later on, Ostieri and Tognaccini [14] pointed out that this definition tends to systematically overestimate the wave drag. Hence, they used Gauss theorem in order to transform the surface integral Eq. (7) into a volume integral limited to the boundary 
layer and shock wave regions $\Omega_{v}$ and $\Omega_{w}$. It was surmised that the volume $\Omega_{w}$ should contain the Lamb vector field produced in the near wake of the shock in order to correctly compute the wave drag [14].

Hence, the Lamb vector based decomposition is very promising since it relies on an exact formula and might well predict all the contributions of the drag along with the lift. However, there are still flaws to tackle in order to enhance the robustness of the method:

- The dependence on the reference point adopted for the computation of moments.

- The sensitivity to the integration domain.

- A robust wave drag definition.

\section{The dependence on the reference point of the aerodynamic force breakdown}

\section{A. The limitations of the present formulation}

The surface integral $\vec{F}_{S_{e}}$ can be limited to the wake where the Lamb vector $\vec{l}$ is not zero: in practice, the abovementioned authors adopted a wake plane perpendicular to the freestream velocity $\vec{U}_{\infty}=U_{\infty} \vec{e}_{x}$ for the computation of the profile drag. To do so, one must first proceed to an interpolation of the numerical solution onto this plane and may lose accuracy. Furthermore, the use of derivative moment transformations Eq. (46) and Eq.(47) introduce the position vector $\vec{r}$ in Eq. (6) and Eq. (7). The total force formula (4) is indeed independent of the reference point but the individual terms therein depend on its choice which raises the question: is the force decomposition dependent on the reference point adopted for the computation of moments? In other words, is the breakdown likely to be changed if one chooses a reference point different from the origin of the frame?

One way to circumvent this issue has hitherto consisted in setting this point on the wake plane adopted for profile drag computation [10, 13, 15, 16], which also reduces $\vec{F}_{S_{e}}$ to a drag contribution only. However, when $\vec{r}$ is shifted by a constant $\vec{r}_{0}$ (i.e. $\vec{r} \rightarrow \vec{r}+\vec{r}_{0}$ ) in $\vec{F}_{S_{e}}$ and $\vec{F}_{m_{\rho}}$, an additional term is generated:

$$
\begin{aligned}
\vec{F}_{S_{e}} & \rightarrow \vec{F}_{S_{e}}-\frac{\vec{r}_{0}}{n-1} \times \int_{S_{e}} \vec{n} \times \rho \vec{l} \mathrm{~d} S \\
\vec{F}_{m_{\rho}} & \rightarrow \vec{F}_{m_{\rho}}+\frac{\vec{r}_{0}}{n-1} \times \int_{S_{e}} \vec{n} \times \frac{u^{2}}{2} \nabla \rho \mathrm{d} S
\end{aligned}
$$

Then, the contribution to the total force is changed which makes it tough to correctly define the different drag components.

\section{B. Profile drag computation for various positions of the reference point}

A numerical computation was performed using RANS equations, Spalart-Allmaras turbulence model and Jameson's scheme (cell-centered, $2^{\text {nd }}$ and $4^{\text {th }}$ order artificial viscosities) in the AIRBUS-SAFRAN-ONERA elsA solver for a transonic steady viscous flow around an OAT15A airfoil (see Fig 2) with a Mach number $M_{\infty}=0.724$, a Reynolds number $R e=3 \times 10^{6}$ and an incidence $\alpha=1.15^{\circ}$. The numerical solution was then postprocessed with a FORTRAN code in order to compute the aerodynamic force acting on the airfoil. The external boundary $S_{e}$ is defined by the distance $d$ between the nodes where integration is performed and the body surface $S_{b}$ as sketched in Fig $2 \mathrm{~b}$.

The evolution of the profile drag with respect to the distance $d$ for various $\left(X_{0}, Z_{0}\right)$-locations of the reference point is sketched in Fig 3 It is clear that changing the reference point leads to significant disparities in the assessment of the profile drag when $S_{e}$ is placed close to the airfoil. Nevertheless, those figures also highlight that these disparities vanish as one marches further downstream in the wake.

Therefore it is of high interest to identify the mechanisms that lead to a reference point invariant aerodynamic force breakdown. According to Eq.99, the behaviour exhibited in Fig 3 suggests that the far field satisfies:

$$
\int_{S_{e}} \vec{n} \times \rho \vec{l} \mathrm{~d} S \approx \overrightarrow{0}
$$

This condition is actually fulfilled in the far wake since, as one marches downstream the wake spreads out because of viscous diffusion and progressively satisfies symmetry properties with respect to the $(x, y)$-plane. 


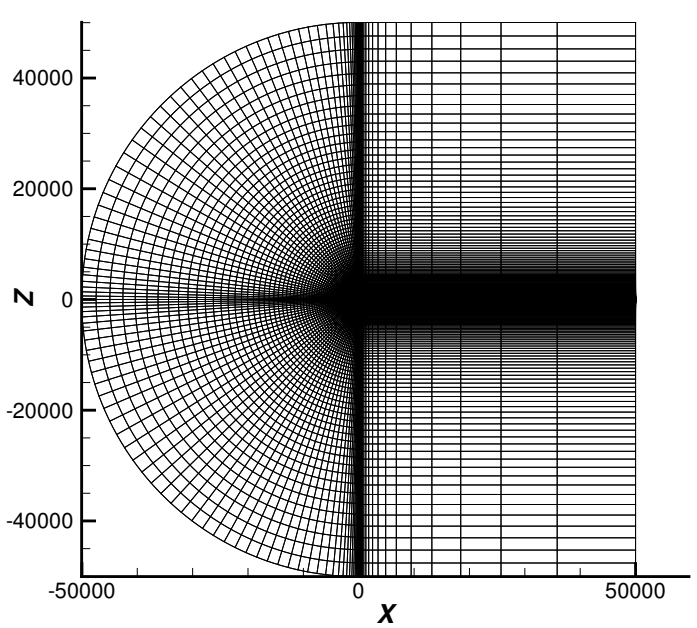

(a) H-C type mesh with two blocks (1 point over 4 )

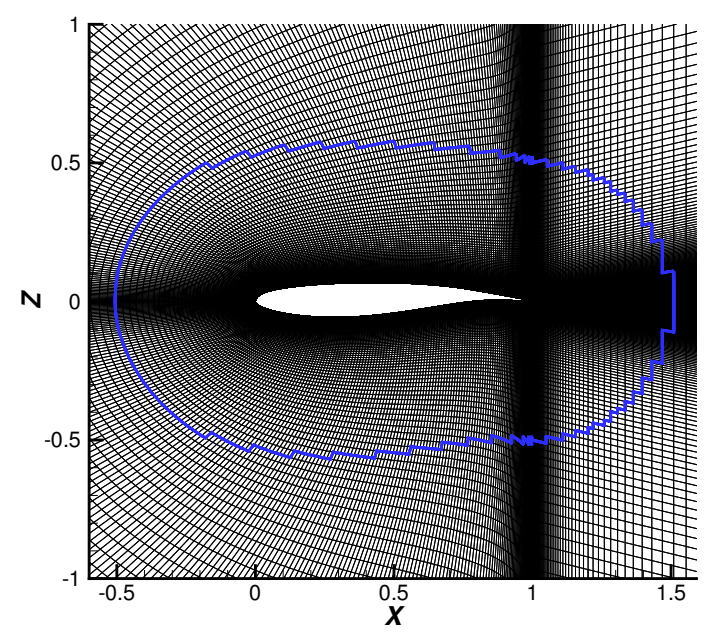

(b) Near-wall mesh resolution $\left(S_{e}\right.$ in blue)

Fig. 2 OAT15A airfoil grid

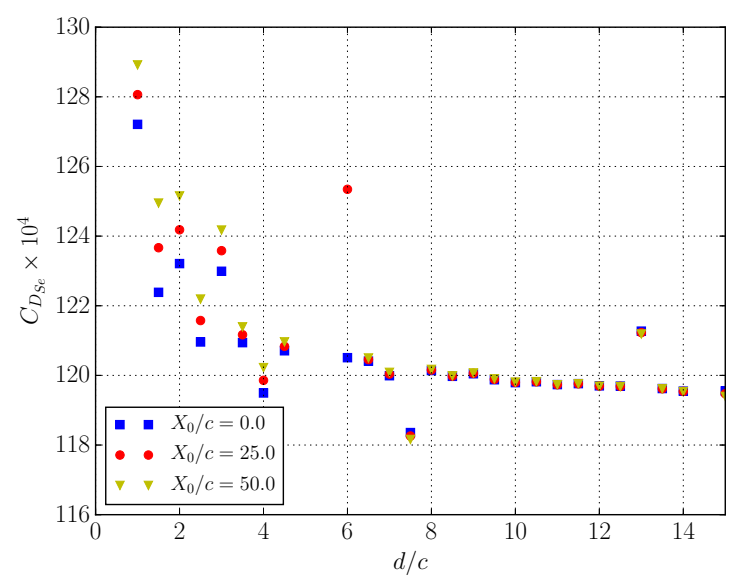

(a) $X \rightarrow X+X_{0}$

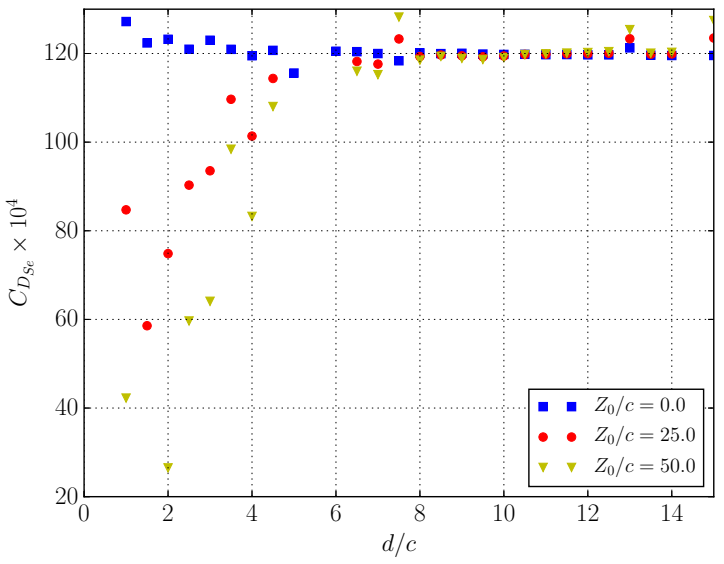

(b) $Z \rightarrow Z+Z_{0}$

Fig. $3 C_{D_{S_{e}}}$ variations for the OAT15A, $M_{\infty}=0.724, R e=3 \times 10^{6}$ and $\alpha=1.15^{\circ}$

\section{The flow symmetries}

\section{A. A simplified two-dimensional case}

To illustrate this point with a simple example, consider the two-dimensional flow described above and assume that the surface integration is performed on a wake plane $\Sigma$ with $\vec{n}=\vec{e}_{x}$ aligned with the freestream direction (see Fig $1 \mathrm{~b}$ ). In this case:

$$
\begin{aligned}
\int_{\Sigma} \vec{n} \times \rho \vec{l} \mathrm{~d} S & =\left(\int_{-z_{\infty}}^{z_{\infty}} \rho l_{z} \mathrm{~d} z\right) \vec{e}_{y} \\
\int_{\Sigma} \vec{n} \times \frac{u^{2}}{2} \nabla \rho \mathrm{d} S & =\left(\int_{-z_{\infty}}^{z_{\infty}} \frac{u^{2}}{2} \frac{\partial \rho}{\partial z} \mathrm{~d} z\right) \vec{e}_{y}
\end{aligned}
$$




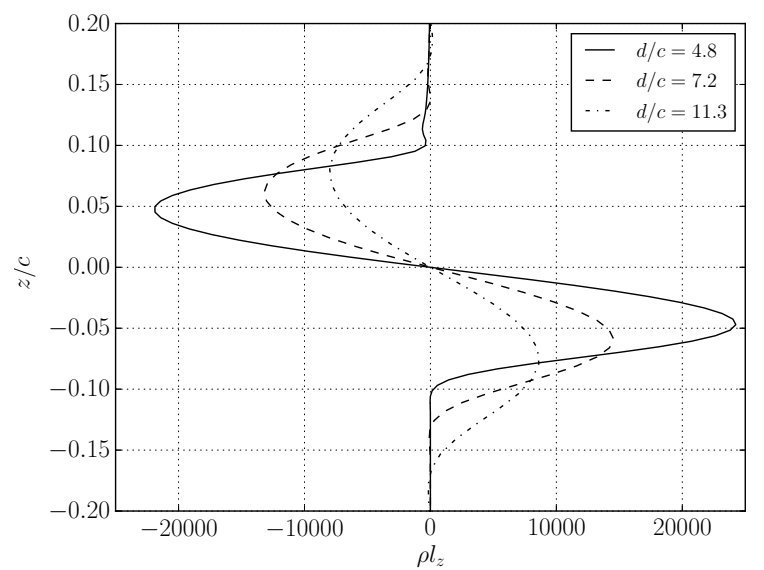

(a) $\rho l_{z}$ profile with respect to $z$

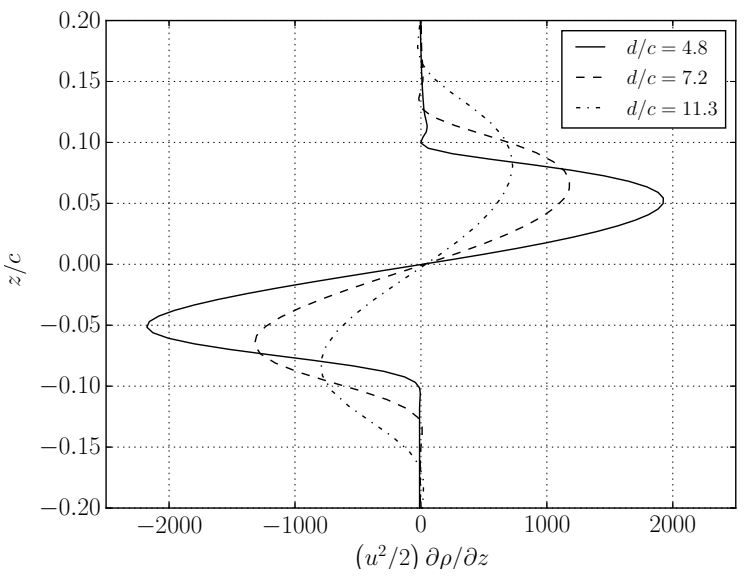

(b) $\frac{u^{2}}{2} \frac{\partial \rho}{\partial z}$ profile with respect to $z$

Fig. 4 2D wake evolution of $\rho l_{z}$ and $\frac{u^{2}}{2} \frac{\partial \rho}{\partial z}$

Fig 4 displays the evolution of the $\rho l_{z}$ and $\frac{u^{2}}{2} \frac{\partial \rho}{\partial z}$ profiles with respect to $z$ for various positions of the wake plane. As one marches further downstream, the region where $\rho l_{z} \neq 0$ and $\frac{u^{2}}{2} \frac{\partial \rho}{\partial z} \neq 0$ expands due to the spread of the wake and there exists a $z$-position for which the profiles become gradually antisymmetrical. Hence, in the case of a two-dimensional flow with a wake plane $\Sigma$ taken perpendicular to the freestream direction $\vec{e}_{x}$, Eqs.(12) and (13) yield

$$
\begin{aligned}
& \lim _{d \rightarrow \infty} \int_{\Sigma} \vec{n} \times \rho \vec{l} \mathrm{~d} S=\overrightarrow{0} \\
& \lim _{d \rightarrow \infty} \int_{\Sigma} \vec{n} \times \frac{u^{2}}{2} \nabla \rho \mathrm{d} S=\overrightarrow{0}
\end{aligned}
$$

such that the aerodynamic force breakdown progressively becomes independent of the reference point in the far wake.

\section{B. The three-dimensional wake flow symmetries}

The study of symmetries is widely spread in modern physics since they are often used to shed light on invariance properties. Under steady cruise flight conditions, it is expected that the flow around an aircraft naturally satisfy a symmetry with respect to the $(x, z)$-plane. Further downstream, the velocity defect $u_{x}-U_{\infty}$ caused by the boundary layers and shock waves progressively becomes symmetrical with respect to the $(x, y)$-plane [17] because of the rollingup of the vortex sheet and the viscous diffusion of the wake. Indeed, the rolling-up of the trailing vortex sheet gives birth to a pair of counter-rotating vortices [18-20] as sketched in Fig 5 From now on, the focus is set on the steady far field [21, 22] where the expansion of the wake and the trailing vortices is assumed to be slow.

Thus, far enough downstream, the symmetry properties that are presented below are based on the concentrated dipole [19, 23] sketched in Fig 5 and the following assumptions:

1) The longitudinal velocity distribution is symmetrical with respect to the $(x, y)$ and $(x, z)$-planes.

2) The transverse part of the flow field consists of a counter-rotating vortex pair as observed by Misaka et al. [20].

3) The $x$-derivatives are negligible compared to the $y$ and $z$-derivatives [24]: $\frac{\partial}{\partial x} \ll \frac{\partial}{\partial y}, \frac{\partial}{\partial z}$.

4) The external boundary $S_{e}$ is assumed to be symmetrical with respect to the $(x, y),(x, z)$ and $(y, z)$-planes.

Let $\overrightarrow{\vec{S}}_{x}, \overrightarrow{\vec{S}}_{y}$ and $\overrightarrow{\vec{S}}_{z}$ be defined as follows:

$$
\overrightarrow{\vec{S}}_{x}=\left(\begin{array}{ccc}
-1 & 0 & 0 \\
0 & 1 & 0 \\
0 & 0 & 1
\end{array}\right), \quad \overrightarrow{\vec{S}}_{y}=\left(\begin{array}{ccc}
1 & 0 & 0 \\
0 & -1 & 0 \\
0 & 0 & 1
\end{array}\right) \quad \text { and } \quad \overrightarrow{\vec{S}}_{z}=\left(\begin{array}{ccc}
1 & 0 & 0 \\
0 & 1 & 0 \\
0 & 0 & -1
\end{array}\right)
$$




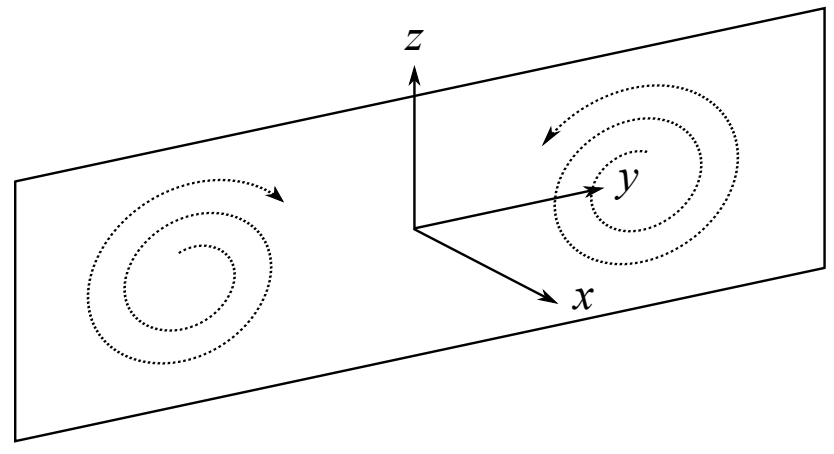

The first two hypotheses imply that the velocity field and the density satisfy (from now on $\Phi=\frac{u^{2}}{2}$ or $\rho$ ):

$$
\begin{aligned}
\vec{u}(x,-y, z) & =\overrightarrow{\vec{S}}_{y} \cdot \vec{u}(x, y, z) \\
\vec{u}(x, y,-z) & =\overrightarrow{\vec{S}}_{y} \cdot \vec{u}(x, y, z) \\
\Phi(x,-y, z) & =\Phi(x, y, z) \\
\Phi(x, y,-z) & =\Phi(x, y, z)
\end{aligned}
$$

\section{Fig. 5 Counter-rotating vortex pair in the far wake}

Say that a scalar function $f: x \rightarrow f(x)$ fulfills $f(-x)=f(x)$, therefore its derivative satisfies $f^{\prime}(-x)=-f^{\prime}(x)$. Upon applying this property to the velocity vector field by using Eqs. (17a), (17b) and (50), it is possible to prove that the vorticity vector field verifies:

$$
\left\{\begin{array} { l } 
{ \omega _ { x } ( x , - y , z ) = - \omega _ { x } ( x , y , z ) } \\
{ \omega _ { y } ( x , - y , z ) = \omega _ { y } ( x , y , z ) } \\
{ \omega _ { z } ( x , - y , z ) = - \omega _ { z } ( x , y , z ) }
\end{array} \quad \text { and } \quad \left\{\begin{array}{l}
\omega_{x}(x, y,-z)=\omega_{x}(x, y, z) \\
\omega_{y}(x, y,-z)=-\omega_{y}(x, y, z)+x \text {-derivatives } \\
\omega_{z}(x, y,-z)=\omega_{z}(x, y, z)+x \text {-derivatives }
\end{array}\right.\right.
$$

Under the third hypothesis, one may write the following symmetry properties for the vorticity field:

$$
\begin{aligned}
& \vec{\omega}(x,-y, z)=-\overrightarrow{\vec{S}}_{y} \cdot \vec{\omega}(x, y, z) \\
& \vec{\omega}(x, y,-z)=\overrightarrow{\vec{S}}_{y} \cdot \vec{\omega}(x, y, z)
\end{aligned}
$$

Therefore, using Eqs.(17a), (17b), 17c), (17d), 19a) and (19b) and knowing that $\vec{l}=\vec{\omega} \times \vec{u}$, the symmetry properties of the Lamb vector field in the far wake write:

$$
\begin{aligned}
& \rho \vec{l}(x,-y, z)=\overrightarrow{\vec{S}}_{y} \cdot \rho \vec{l}(x, y, z) \\
& \rho \vec{l}(x, y,-z)=-\overrightarrow{\vec{S}}_{y} \cdot \rho \vec{l}(x, y, z)
\end{aligned}
$$

Besides, using Eqs. 17c), (17d), 48] and 49] one may express the symmetry properties satisfied by $\frac{u^{2}}{2} \nabla \rho$ :

$$
\begin{aligned}
& \frac{u^{2}}{2} \nabla \rho(x,-y, z)=\overrightarrow{\vec{S}}_{y} \cdot \frac{u^{2}}{2} \nabla \rho(x, y, z) \\
& \frac{u^{2}}{2} \nabla \rho(x, y,-z)=\overrightarrow{\vec{S}}_{z} \cdot \frac{u^{2}}{2} \nabla \rho(x, y, z)
\end{aligned}
$$

The fourth hypothesis implies that the unit normal is symmetrical with respect to the $(x, y)$-plane and the $(x, z)$-plane:

$$
\begin{aligned}
& \vec{n}(x,-y, z)=\overrightarrow{\vec{S}}_{y} \cdot \vec{n}(x, y, z) \\
& \vec{n}(x, y,-z)=\overrightarrow{\vec{S}}_{z} \cdot \vec{n}(x, y, z)
\end{aligned}
$$

In the end, the symmetry properties derived above yield:

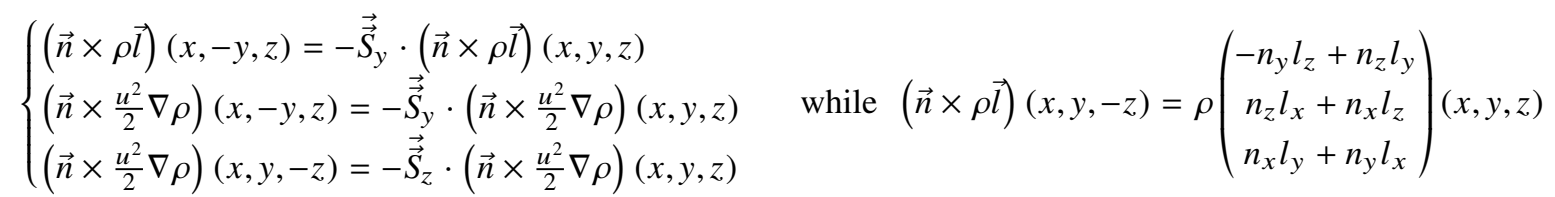


Let $S_{w}$ be the portion of $S_{e}$ crossing the wake. One can now integrate those vectors on $S_{w}$ such that:

$$
\begin{aligned}
\int_{S_{w}}\left(\vec{n} \times \frac{u^{2}}{2} \nabla \rho\right) \mathrm{d} S & =\int_{S_{w}, y \geq 0}\left(\vec{n} \times \frac{u^{2}}{2} \nabla \rho\right) \mathrm{d} S+\int_{S_{w}, y \leq 0}\left(\vec{n} \times \frac{u^{2}}{2} \nabla \rho\right) \mathrm{d} S \\
& =\left(\overrightarrow{\overrightarrow{1}}-\overrightarrow{\vec{S}}_{y}\right) \cdot \int_{S_{w}, y \geq 0}\left(\vec{n} \times \frac{u^{2}}{2} \nabla \rho\right) \mathrm{d} S \\
& =\underbrace{\left(\overrightarrow{\overrightarrow{1}}-\overrightarrow{\vec{S}}_{y}\right) \cdot\left(\overrightarrow{\overrightarrow{1}}-\overrightarrow{\vec{S}}_{z}\right)}_{0} \cdot \int_{S_{w}, y, z \geq 0}\left(\vec{n} \times \frac{u^{2}}{2} \nabla \rho\right) \mathrm{d} S=\overrightarrow{0}
\end{aligned}
$$

while

$$
\begin{aligned}
\int_{S_{w}}(\vec{n} \times \rho \vec{l}) \mathrm{d} S & =\left(\overrightarrow{\overrightarrow{1}}-\overrightarrow{\vec{S}}_{y}\right) \cdot \int_{S_{w}, y \geq 0}(\vec{n} \times \rho \vec{l}) \mathrm{d} S \\
& =\left(\overrightarrow{\overrightarrow{1}}-\overrightarrow{\vec{S}}_{y}\right) \cdot \int_{S_{w}, y, z \geq 0} \rho\left[\left(\begin{array}{l}
n_{y} l_{z}-n_{z} l_{y} \\
n_{z} l_{x}-n_{x} l_{z} \\
n_{x} l_{y}-n_{y} l_{x}
\end{array}\right)+\left(\begin{array}{c}
-n_{y} l_{z}+n_{z} l_{y} \\
n_{z} l_{x}+n_{x} l_{z} \\
n_{x} l_{y}+n_{y} l_{x}
\end{array}\right)\right] \mathrm{d} S \\
& =2\left(\overrightarrow{\overrightarrow{1}}-\overrightarrow{\vec{S}}_{y}\right) \cdot \int_{S_{w}, y, z \geq 0} \rho\left(\begin{array}{c}
0 \\
n_{z} l_{x} \\
n_{x} l_{y}
\end{array}\right) \mathrm{d} S=4 \int_{S_{w}, y, z \geq 0} \rho\left(\begin{array}{c}
0 \\
n_{z} l_{x} \\
0
\end{array}\right) \mathrm{d} S
\end{aligned}
$$

Thus, Eq. 25] suggests that $\int_{S_{w}}(\vec{n} \times \rho \vec{l}) \mathrm{d} S \neq \overrightarrow{0}$. Nevertheless, in the far wake the velocity field can be approximated as $\vec{u}=\left(U_{\infty}+u_{x}^{\prime}\right) \vec{e}_{x}+u_{y} \vec{e}_{y}+u_{z} \vec{e}_{z}$ such that $\left|\frac{u_{x}^{\prime}}{U_{\infty}}\right|,\left|\frac{u_{y}}{U_{\infty}}\right|,\left|\frac{u_{z}}{U_{\infty}}\right| \ll 1$. In this case, the Lamb vector consists of a first order and a second order term:

$$
\vec{l}=\underbrace{\left(\begin{array}{c}
0 \\
\omega_{z} U_{\infty} \\
-\omega_{y} U_{\infty}
\end{array}\right)}_{\text {first order }}+\underbrace{\left(\begin{array}{l}
\omega_{y} u_{z}-\omega_{z} u_{y} \\
\omega_{z} u_{x}^{\prime}-\omega_{x} u_{z} \\
\omega_{x} u_{y}-\omega_{y} u_{x}^{\prime}
\end{array}\right)}_{\text {second order }}
$$

Besides, in the far wake the direction of the flow is almost parallel to that of the freestream and the region where the Lamb vector is not zero coincides with a portion of $S_{w}$ where $\vec{n} \approx \vec{e}_{x}$. Therefore, at first order $l_{x} \ll l_{y}, l_{z}$ and $n_{y}, n_{z} \ll n_{x}$ such that the integral Eq. 25$]$ becomes negligible (of the order of magnitude $10^{-}$as shown in

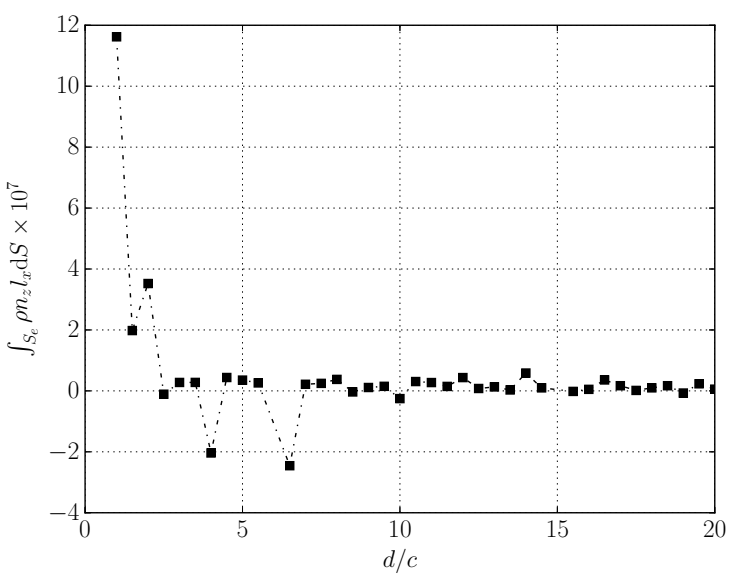

Fig. 6 Evolution of the integral Eq. 25 in the wake Fig 6) and the condition Eq.(11) is progressively verified.

\section{The upstream-downstream symmetry properties}

The study presented above focused on the symmetries satisfied in the wake of an airfoil. Indeed, the Lamb vector field is not zero only in the boundary layers, in the wake and behind a curved shock wave. On the contrary, $\frac{u^{2}}{2} \nabla \rho$ may well be different from zero in other regions of the flow. For instance, in compressible flows the presence of an obstacle produces a density gradient further upstream of the leading edge and as the flow marches downstream of the obstacle, the density progressively recovers its freestream value.

To illustrate this point, numerical computations were performed using RANS equations and Spalart-Allmaras turbulence model for a compressible steady viscous flow around a NACA0012 airfoil with a Mach number $M_{\infty}=0.4$ and a Reynolds number $R e=3 \times 10^{6}$. Fig 7 sketches contours of $\frac{u^{2}}{2} \nabla \rho$ for $\alpha=0^{\circ}$ and $\alpha=1.15^{\circ}$. In the symmetrical case (see Figs $7 \mathrm{a}$ and $7 \mathrm{~b}$ ), the flow first undergoes a compression in front of the leading edge which is characterized by a positive $x$-wise density gradient. It further accelerates on the pressure and suction sides because of the wall curvature 


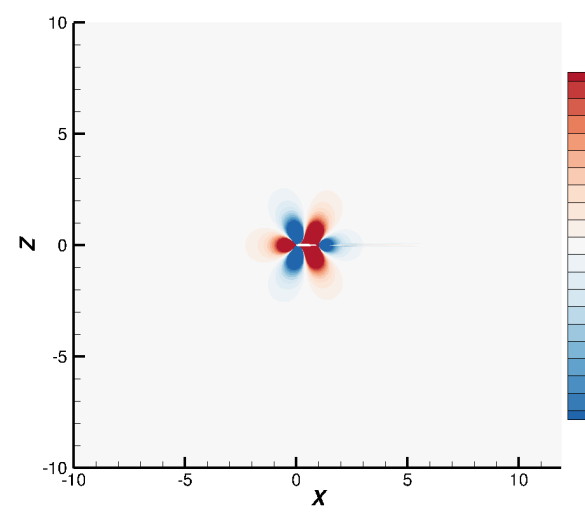

(a) Contour of $\frac{u^{2}}{2} \frac{\partial \rho}{\partial x}$ for $\alpha=0^{\circ}$

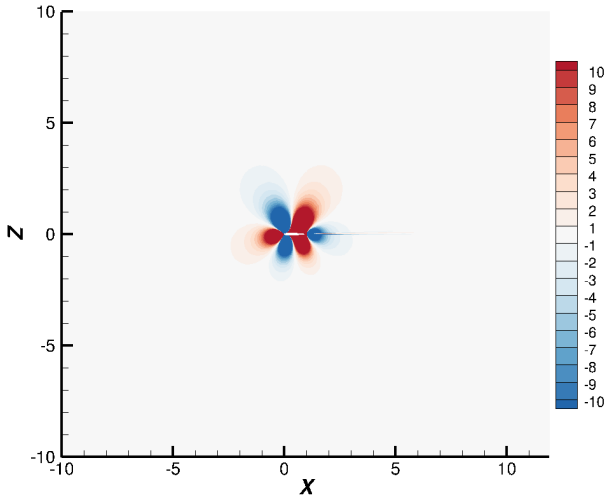

(c) Contour of $\frac{u^{2}}{2} \frac{\partial \rho}{\partial x}$ for $\alpha=1.15^{\circ}$

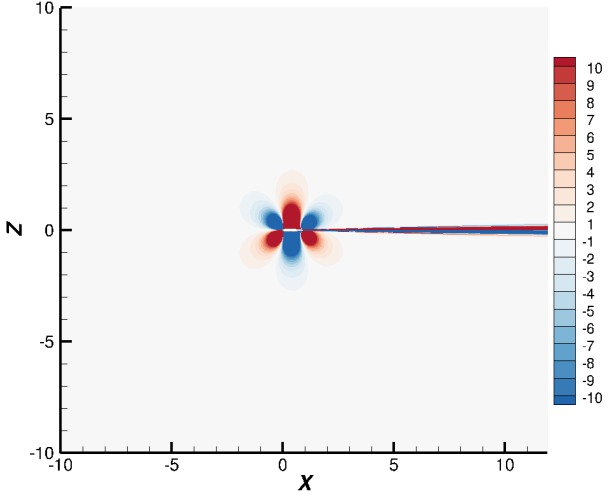

(b) Contour of $\frac{u^{2}}{2} \frac{\partial \rho}{\partial z}$ for $\alpha=0^{\circ}$

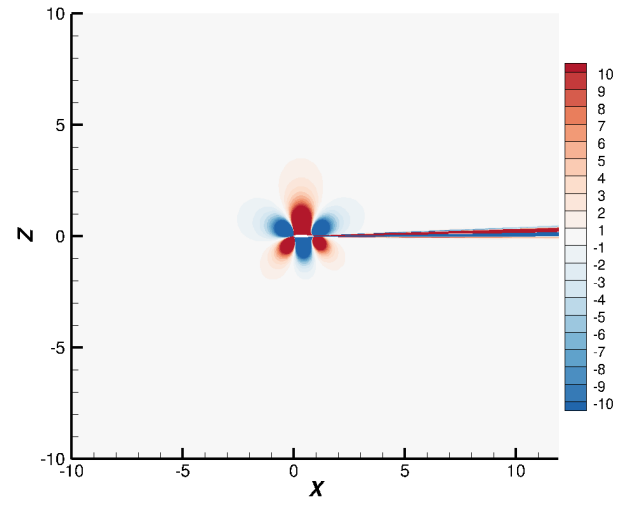

(d) Contour of $\frac{u^{2}}{2} \frac{\partial \rho}{\partial z}$ for $\alpha=1.15^{\circ}$

Fig. 7 Contours of $\frac{u^{2}}{2} \nabla \rho$ around a NACA0012 airfoil at $R e=3 \times 10^{6}, M_{\infty}=0.4$ with and without incidence

which yields a negative $x$-wise density gradient (expansion). Further downstream the flow faces an adverse pressure gradient and decelerates: it corresponds to the region where the $x$-wise density gradient becomes positive. Downstream of the trailing edge the flow is again expanded and the $x$-wise density gradient becomes negative. It is noteworthy that the flow satisfies a symmetry with respect to the $(x, y)$-plane since the airfoil itself is symmetrical and $\alpha=0^{\circ}$. The above-described phenomena also occur when $\alpha=1.15^{\circ}$ (see Figs.7c and $7 \mathrm{~d}$ ) but the symmetry observed in the symmetrical case vanishes: indeed, this symmetry breaking is due to the generation of lift. Outside the wake, the study of the far field suggests that $\frac{u^{2}}{2} \nabla \rho$ fulfills the following symmetry property with respect to the $(y, z)$-plane as shown in Figs 7 and 8 .

$$
\frac{u^{2}}{2} \nabla \rho(-x, y, z)=\overrightarrow{\vec{S}}_{x} \cdot \frac{u^{2}}{2} \nabla \rho(x, y, z)
$$

Moreover, by using the fourth hypothesis the unit normal satisfies:

$$
\vec{n}(-x, y, z)=\overrightarrow{\vec{S}}_{x} \cdot \vec{n}(x, y, z)
$$

such that:

$$
\left(\vec{n} \times \frac{u^{2}}{2} \nabla \rho\right)(-x, y, z)=-\overrightarrow{\vec{S}}_{x} \cdot\left(\vec{n} \times \frac{u^{2}}{2} \nabla \rho\right)(x, y, z)
$$


Hence, the following condition is derived:

$$
\int_{S_{e}-S_{w}}\left(\vec{n} \times \frac{u^{2}}{2} \nabla \rho\right) \mathrm{d} S=\underbrace{\left(\overrightarrow{\overrightarrow{1}}-\overrightarrow{\vec{S}}_{x}\right) \cdot\left(\overrightarrow{\overrightarrow{1}}-\overrightarrow{\vec{S}}_{y}\right)}_{0} \cdot \int_{S_{e}-S_{w}, x, y \geq 0}\left(\vec{n} \times \frac{u^{2}}{2} \nabla \rho\right) \mathrm{d} S=\overrightarrow{0}
$$

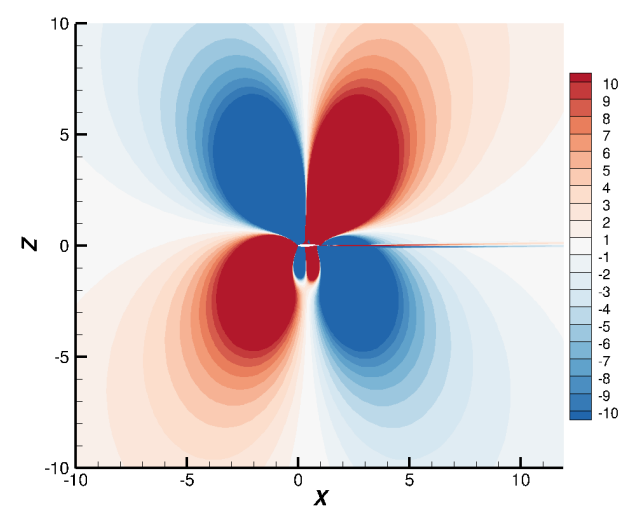

(a) Contour of $\frac{u^{2}}{2} \frac{\partial \rho}{\partial x}$

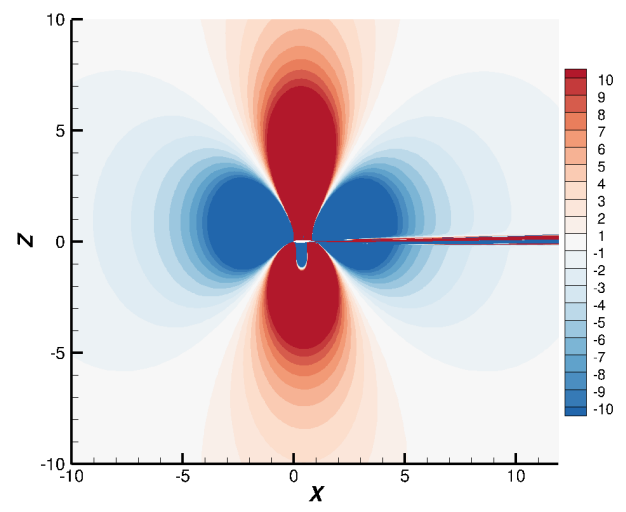

(b) Contour of $\frac{u^{2}}{2} \frac{\partial \rho}{\partial z}$

Fig. 8 Contours of $\frac{u^{2}}{2} \nabla \rho$ around an OAT15A airfoil at $R e=3 \times 10^{6}, M_{\infty}=0.724$ and $\alpha=1.15^{\circ}$

Therefore, the three-dimensional air flow around an object which is symmetrical with respect to the $(x, z)$-plane will gradually satisfy symmetry properties on $S_{e}$ as the size of the control volume increases such that the Lamb vector based aerodynamic force breakdown is independent of the reference point in the far field:

$$
\begin{aligned}
& \lim _{d \rightarrow \infty} \int_{S_{e}} \vec{n} \times \rho \vec{l} \mathrm{~d} S=\overrightarrow{0} \\
& \lim _{d \rightarrow \infty} \int_{S_{e}} \vec{n} \times \frac{u^{2}}{2} \nabla \rho \mathrm{d} S=\overrightarrow{0}
\end{aligned}
$$

\section{A reference point invariant formulation}

The study of the flow symmetries highlighted the mechanisms that bring the aerodynamic force decomposition to become independent of the reference point in the far field. Therefore, the control volume must be chosen such that the external boundary $S_{e}$ is placed in the region where the symmetries are fulfilled. Unfortunately, this implies that the grid on which the surface integral is computed becomes ever coarser as the size of the domain is increased. Thus finding a method that would make the formulation independent of the reference point everywhere turns out to be crucial.

\section{A. A systematic method based on symmetries}

To do so, one must fashion vectors based on the physical fields $\rho \vec{l}$ and $\frac{u^{2}}{2} \nabla \rho$ that cancel the additional terms showing up in Eqs.99) and (10) and lead to the same force contribution as the initial vector fields. It consists in extracting the part of the vector field which remains in the far field and satisfies the symmetry properties derived earlier. Let $\phi$ be a function of $\xi$ : then $\phi$ is uniquely decomposed as the sum of a symmetrical part $\phi_{+}$and an antisymmetrical part $\phi_{-}$as follows:

$$
\phi_{+}(\xi)=\frac{1}{2}(\phi(\xi)+\phi(-\xi)) \quad \text { and } \quad \phi_{-}(\xi)=\frac{1}{2}(\phi(\xi)-\phi(-\xi))
$$

Let now $\vec{f}$ be a differentiable vector field and $\vec{F}$ be the associated force contribution defined by Eq.34. The decomposition Eq. 33 can be applied to any of the three components $f_{x}, f_{y}$ and $f_{z}$ with respect to any of the three 
coordinates $x, y$ or $z$. For instance, one can extract $\vec{f}_{x-}$ from $\vec{f}$ for which the $y$ and $z$-components are symmetrical with respect to the $x$-coordinate while its $x$-component is antisymmetrical.

$$
\begin{gathered}
\vec{F}=\frac{1}{n-1} \int_{S_{e}} \vec{r} \times(\vec{n} \times \vec{f}) \mathrm{d} S \\
\vec{f}_{x-}(x, y, z)=\frac{1}{2}\left(\vec{f}(x, y, z)+\overrightarrow{\vec{S}}_{x} \cdot \vec{f}(-x, y, z)\right)
\end{gathered}
$$

In order to comply with the fourth hypothesis, one must integrate this vector field on a surface which satisfies symmetries such that the unit normal fulfills the properties derived above. Then, in the $(x, y)$-plane the variable shift $x^{\prime}=-x$ is performed in the integral containing the second term of $\vec{f}_{x-}$ and Eq.(28) applies. Again, one can extract $\vec{f}_{x y-}$ from $\vec{f}_{x-}$ for which the $x$ and $z$-components are symmetrical with respect to the $y$-coordinate while its $y$-component is antisymmetrical:

$$
\vec{f}_{x y-}(x, y, z)=\frac{1}{4}\left(\vec{f}(x, y, z)+\overrightarrow{\vec{S}}_{x} \cdot \vec{f}(-x, y, z)+\overrightarrow{\vec{S}}_{y} \cdot \vec{f}(x,-y, z)+\overrightarrow{\vec{S}}_{x} \cdot \overrightarrow{\vec{S}}_{y} \cdot \vec{f}(-x,-y, z)\right)
$$

Hence, Eq.22a applies, the cross-product between the unit normal and the vector defined in Eq. (36) satisfies the same $(y, z)$ and $(x, z)$-plane symmetry properties as those derived in the study of the far field:

$$
\begin{aligned}
& \left(\vec{n} \times \vec{f}_{x y-}\right)(-x, y, z)=-\overrightarrow{\vec{S}}_{x} \cdot\left(\vec{n} \times \vec{f}_{x y-}\right)(x, y, z) \\
& \left(\vec{n} \times \vec{f}_{x y-}\right)(x,-y, z)=-\overrightarrow{\vec{S}}_{y} \cdot\left(\vec{n} \times \vec{f}_{x y-}\right)(x, y, z)
\end{aligned}
$$

Therefore the additional term showing up in the surface integral when $\vec{r} \rightarrow \vec{r}+\vec{r}_{0}$ is cancelled while the surface integral of the double cross-product provides the $z$-component of the force contribution:

$$
\begin{gathered}
\int_{S_{e}}\left(\vec{n} \times \vec{f}_{x y-}\right) \mathrm{d} S=\overrightarrow{0} \\
\frac{1}{n-1} \int_{S_{e}} \vec{r} \times\left(\vec{n} \times \vec{f}_{x y-}\right) \mathrm{d} S=\vec{F} \cdot \vec{e}_{z}
\end{gathered}
$$

Similarly, one can define $\vec{f}_{x z-}$ which fulfills the above-written $(y, z)$ and $(x, y)$-plane symmetries and $\vec{f}_{y z-}$ which satisfies the $(x, z)$ and $(x, y)$-plane symmetries such that the additional integrals showing up when $\vec{r} \rightarrow \vec{r}+\vec{r}_{0}$ are cancelled and the surface integral of the double cross-product provide the $x$ and $y$-components of the force contribution:

$$
\begin{gathered}
\int_{S_{e}}\left(\vec{n} \times \vec{f}_{y z^{-}}\right) \mathrm{d} S=\overrightarrow{0} \\
\frac{1}{n-1} \int_{S_{e}} \vec{r} \times\left(\vec{n} \times \vec{f}_{y z^{-}}\right) \mathrm{d} S=\vec{F} \cdot \vec{e}_{x}
\end{gathered}
$$

and

$$
\begin{gathered}
\int_{S_{e}}\left(\vec{n} \times \vec{f}_{x z^{-}}\right) \mathrm{d} S=\overrightarrow{0} \\
\frac{1}{n-1} \int_{S_{e}} \vec{r} \times\left(\vec{n} \times \vec{f}_{x z^{-}}\right) \mathrm{d} S=\vec{F} \cdot \vec{e}_{y}
\end{gathered}
$$

In the end, the force is reconstructed by means of vectors verifying the far field symmetry properties derived earlier everywhere in the control volume such that a shift in the reference point does not change the contribution to the total force, which is by definition independent of the reference point. 


\section{B. The definition of the reference point invariant aerodynamic force breakdown}

The systematic method presented above is now applied to the physical fields $\rho \vec{l}_{\perp}$ and $\frac{u^{2}}{2} \nabla \rho$ with $\vec{l}_{\perp}=l_{y} \vec{e}_{y}+l_{z} \vec{e}_{z}$ defined as the part of the Lamb vector limited to the plane perpendicular to the freestream direction since $l_{x}$ is not involved in the computation of the profile drag and can be neglected in the far wake. Hence, using Eq.46 to cast $\vec{F}_{m_{\rho}}$ as a volume integral plus a surface integral on $S_{e}$, the reference point invariant Lamb vector based aerodynamic force decomposition is defined as follows:

$$
\begin{aligned}
D_{i} & =\vec{e}_{x} \cdot\left\{\frac{1}{n-1} \int_{S_{e}} \vec{r} \times\left(\vec{n} \times \frac{u^{2}}{2} \nabla \rho_{y z^{-}}\right) \mathrm{d} S-\int_{\Omega}\left(\rho \vec{l}-\frac{u^{2}}{2} \nabla \rho\right) \mathrm{d} v\right\} \\
D_{S_{e}} & =\vec{e}_{x} \cdot\left\{-\frac{1}{n-1} \int_{S_{e}} \vec{r} \times\left(\vec{n} \times \rho \vec{l}_{\perp, y z^{-}}\right) \mathrm{d} S\right\} \\
L & =\vec{e}_{z} \cdot\left\{\frac{1}{n-1} \int_{S_{e}} \vec{r} \times\left(\vec{n} \times \frac{u^{2}}{2} \nabla \rho_{x y-}\right) \mathrm{d} S-\int_{\Omega}\left(\rho \vec{l}-\frac{u^{2}}{2} \nabla \rho\right) \mathrm{d} v\right\}
\end{aligned}
$$

\section{Numerical results}

Fig 9 sketches the profile drag computed by the new definition for the two-dimensional transonic flow and must be compared to Fig 3 the curves corresponding to the different shifts in reference point match perfectly which suggests that the new integral is definitely independent of the reference point. Fig 10 shows the drag component of the compressibility

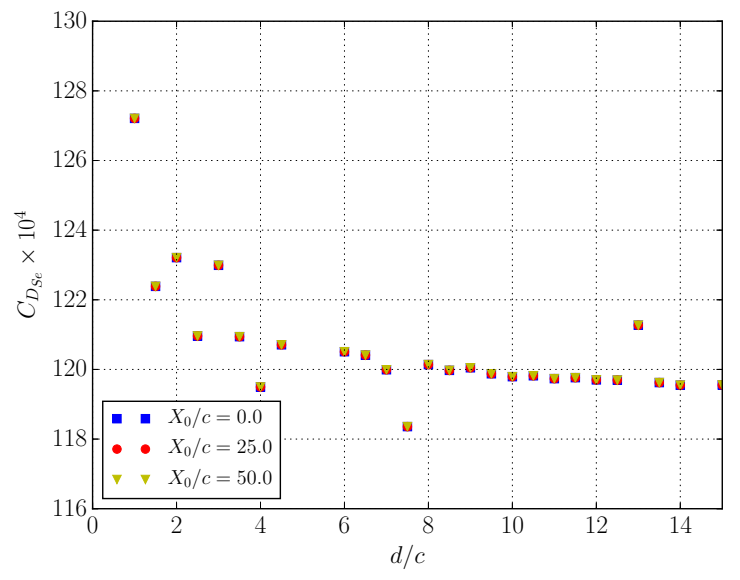

(a) $X \rightarrow X+X_{0}$

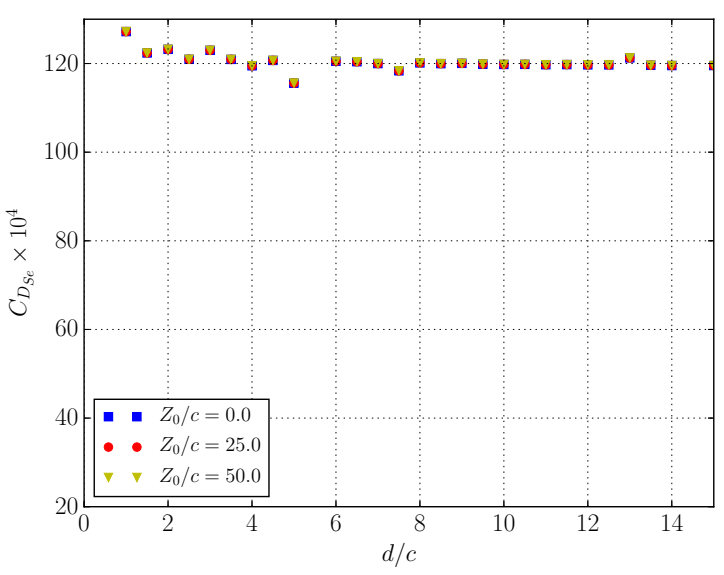

(b) $Z \rightarrow Z+Z_{0}$

Fig. $9 C_{D_{S_{e}}}$ invariance for the OAT15A, $M_{\infty}=0.724, R e=3 \times 10^{6}$ and $\alpha=1.15^{\circ}$

correction term $\vec{F}_{m_{\rho}}$ while Fig 11 sketches the lift component: again one can notice that the new integral is independent of the reference point which suggests that the new formulation guarantees the invariance for two-dimensional cases.

The extension to three-dimensional cases was carried out on the wing of the NASA Common Research Model for a Mach number $M_{\infty}=0.724$, a Reynolds number $R e=3 \times 10^{6}$ and an angle of attack $\alpha=1.15^{\circ}$. Some numerical issues were encountered when computing the lift and lift-induced drag respectively defined by Eq.43) and Eq. (41) (see Figs 13 and 14. Therefore, the invariant form of the term $\vec{F}_{m_{\rho}}$ was computed using the original formula Eq. (6) for which the same method was applied. The lift and the lift-induced drag are then given by:

$$
\begin{aligned}
D_{i} & =\vec{e}_{x} \cdot\left\{-\int_{\Omega} \rho \vec{l} \mathrm{~d} v+\frac{1}{n-1} \int_{\Omega} \vec{r} \times\left(\nabla\left(\frac{u^{2}}{2}\right) \times \nabla \rho\right)_{y z-} \mathrm{d} v\right\} \\
L & =\vec{e}_{z} \cdot\left\{-\int_{\Omega} \rho \vec{l} \mathrm{~d} v+\frac{1}{n-1} \int_{\Omega} \vec{r} \times\left(\nabla\left(\frac{u^{2}}{2}\right) \times \nabla \rho\right)_{x y-} \mathrm{d} v\right\}
\end{aligned}
$$


Figs 12 , 13 and 14 respectively show the computed $C_{D_{S_{e}}}, C_{D_{m_{\rho}}}$ and $C_{L_{m_{\rho}}}$ on the wing of the NASA Common Research Model. The results obtained confirm the invariance of the new formulation for three-dimensional flows.

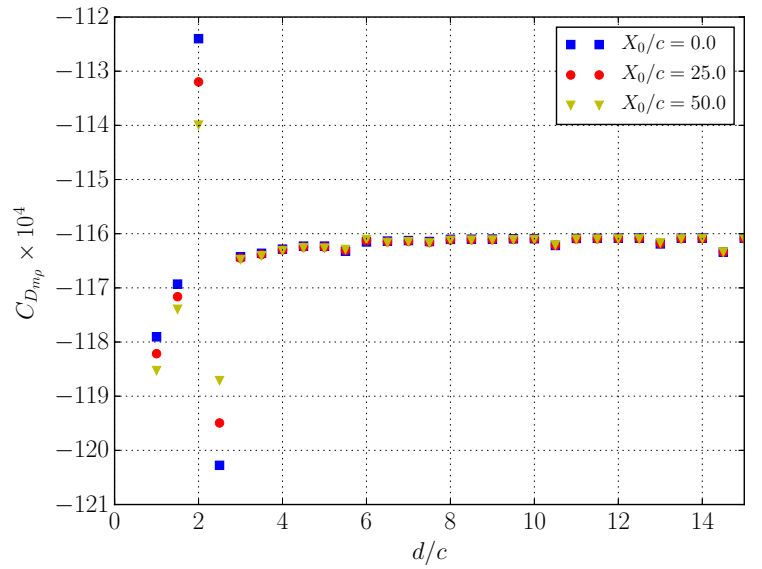

(a) $X \rightarrow X+X_{0}$, old formula

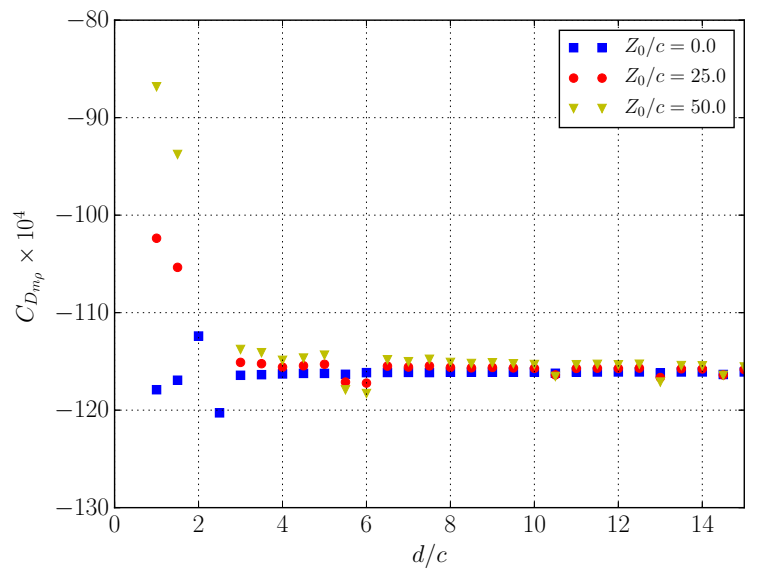

(c) $Z \rightarrow Z+Z_{0}$, old formula

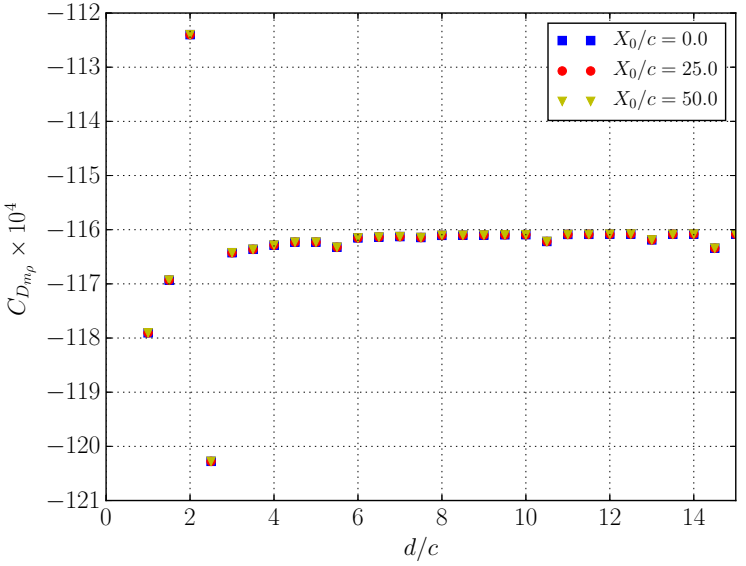

(b) $X \rightarrow X+X_{0}$, new formula

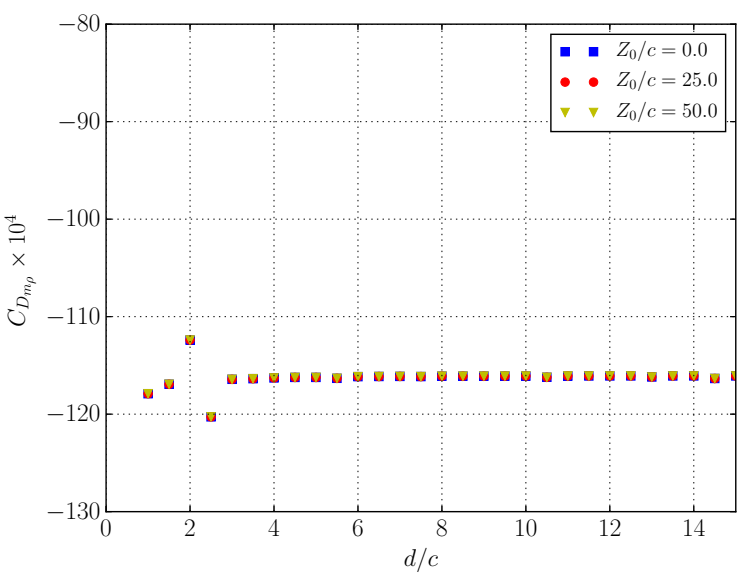

(d) $Z \rightarrow Z+Z_{0}$, new formula

Fig. $10 C_{D_{m_{\rho}}}$ evolutions for the OAT15A, $M_{\infty}=0.724, R e=3 \times 10^{6}$ and $\alpha=1.15^{\circ}$ 


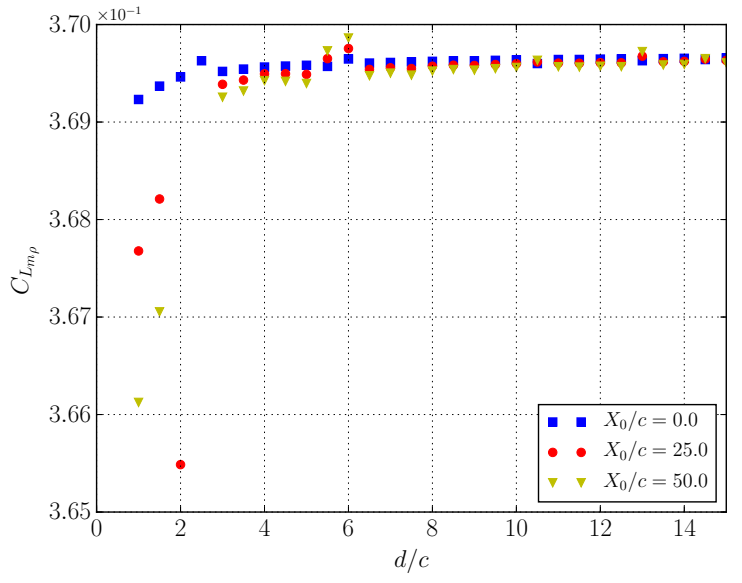

(a) $X \rightarrow X+X_{0}$, old formula

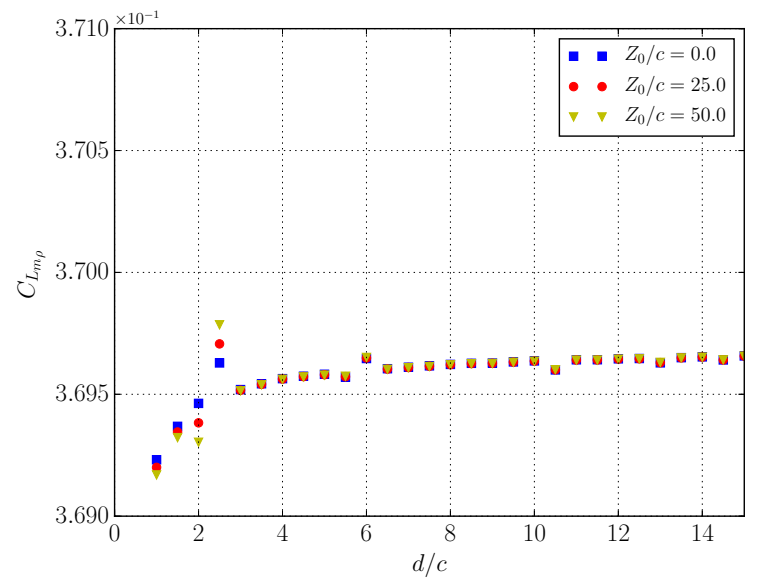

(c) $Z \rightarrow Z+Z_{0}$, old formula

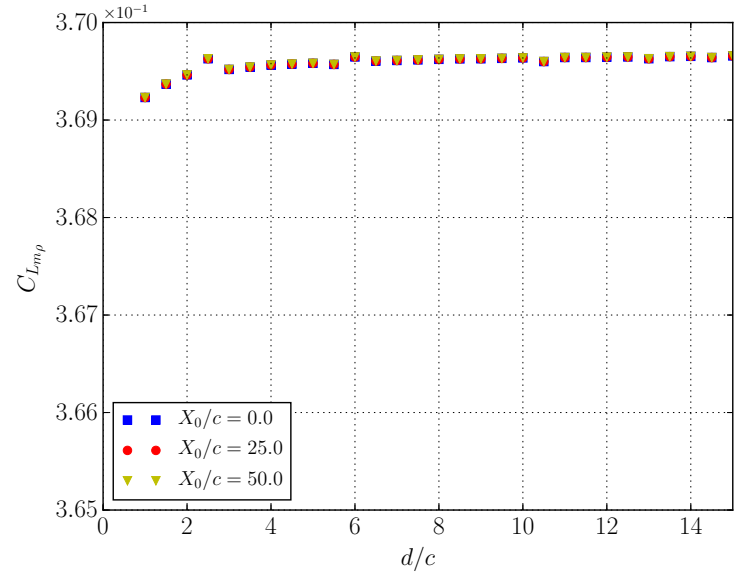

(b) $X \rightarrow X+X_{0}$, new formula

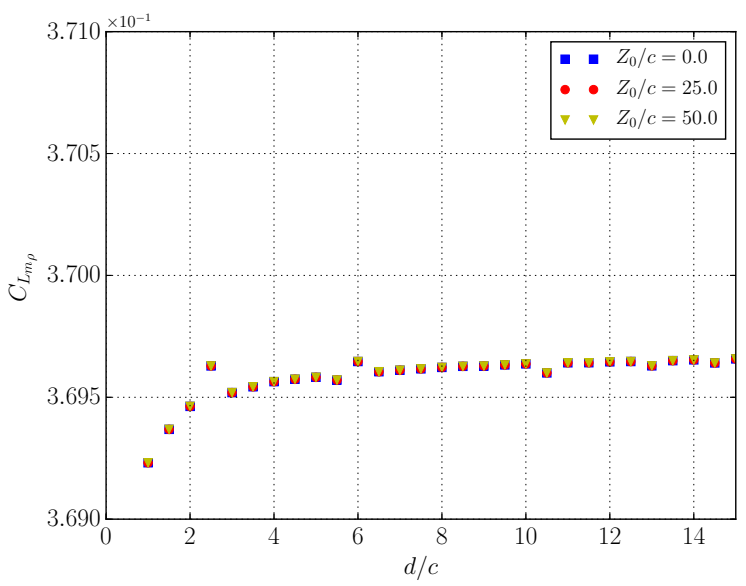

(d) $Z \rightarrow Z+Z_{0}$, new formula

Fig. $11 C_{L_{m_{\rho}}}$ evolutions for the OAT15A, $M_{\infty}=0.724, R e=3 \times 10^{6}$ and $\alpha=1.15^{\circ}$ 


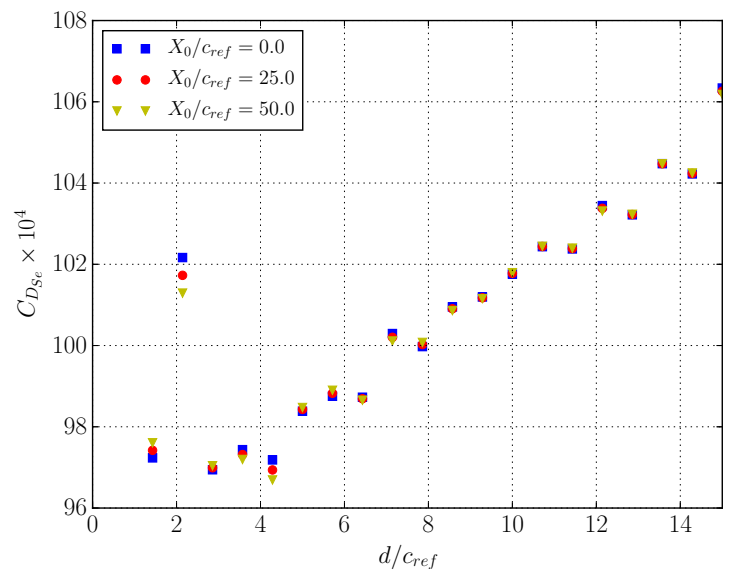

(a) $X \rightarrow X+X_{0}$, old formula

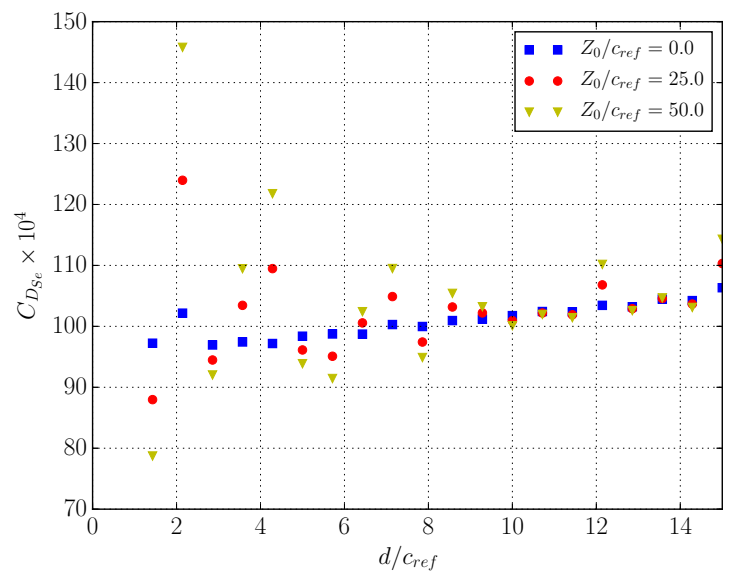

(c) $Z \rightarrow Z+Z_{0}$, old formula

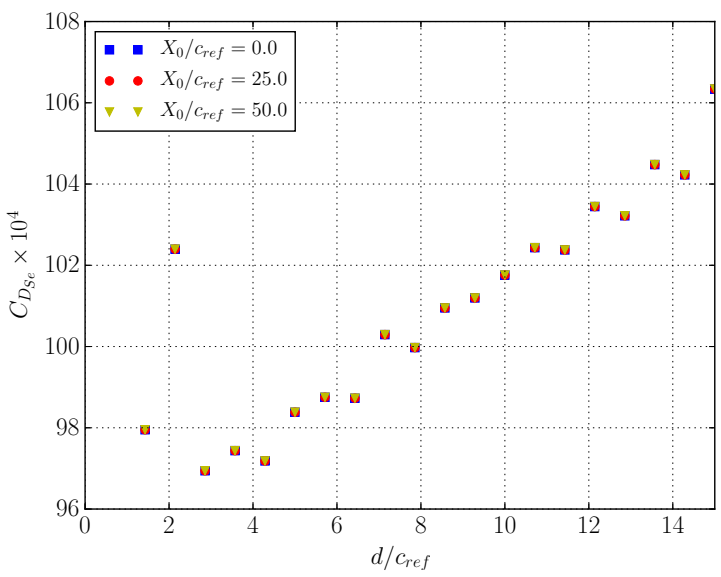

(b) $X \rightarrow X+X_{0}$, new formula

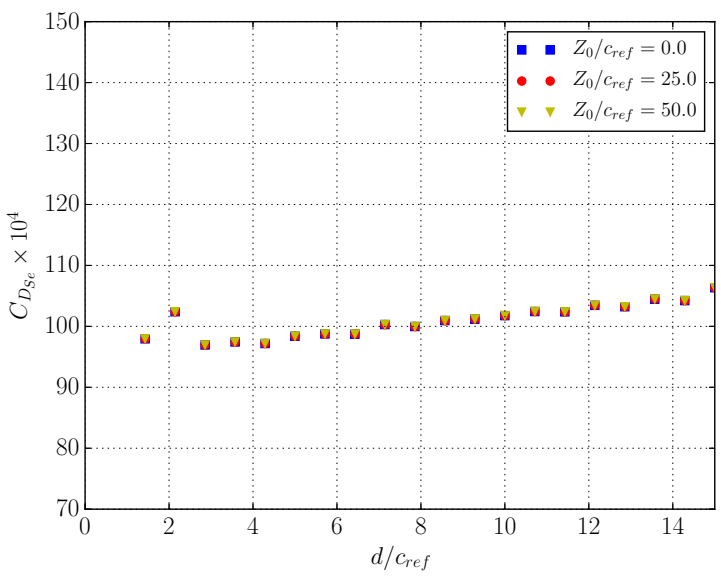

(d) $Z \rightarrow Z+Z_{0}$, new formula

Fig. $12 C_{D_{S e}}$ evolutions for the wing of the NASA CRM, $M_{\infty}=0.724, R e=3 \times 10^{6}$ and $\alpha=1.15^{\circ}$ 


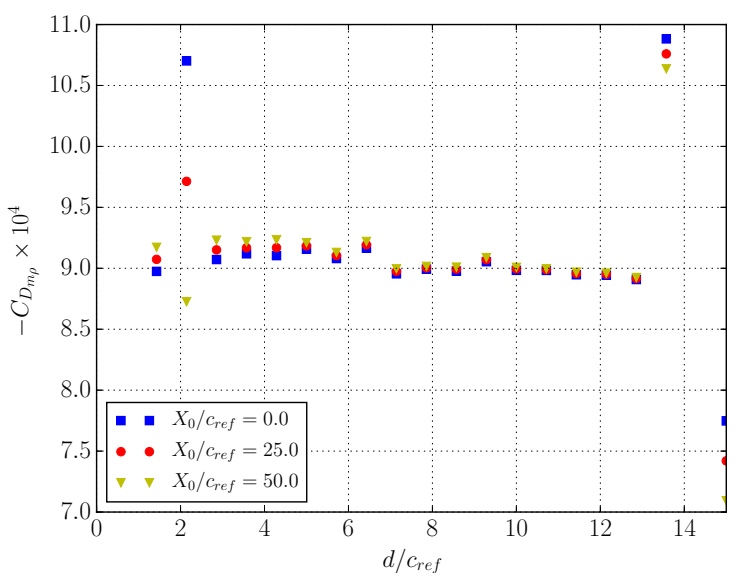

(a) $X \rightarrow X+X_{0}$, old formula

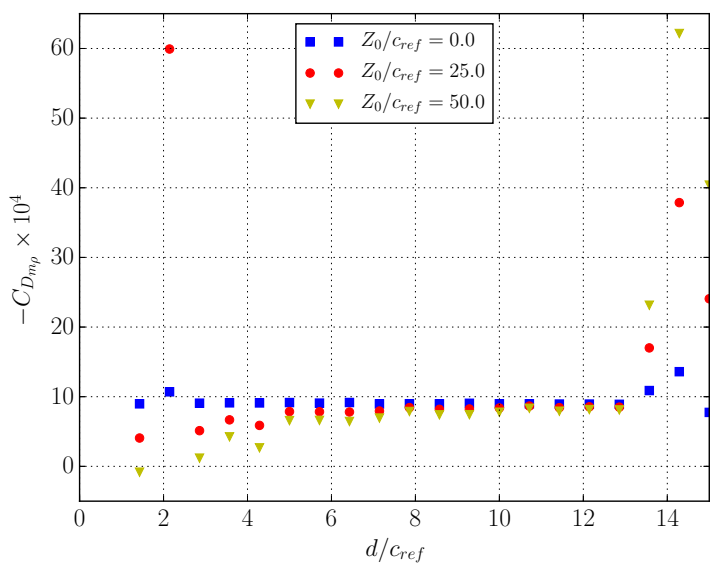

(c) $Z \rightarrow Z+Z_{0}$, old formula

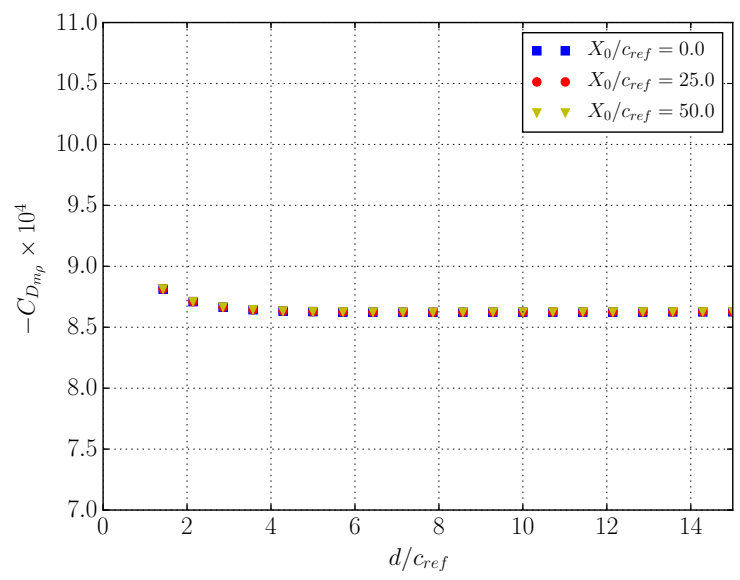

(b) $X \rightarrow X+X_{0}$, new formula

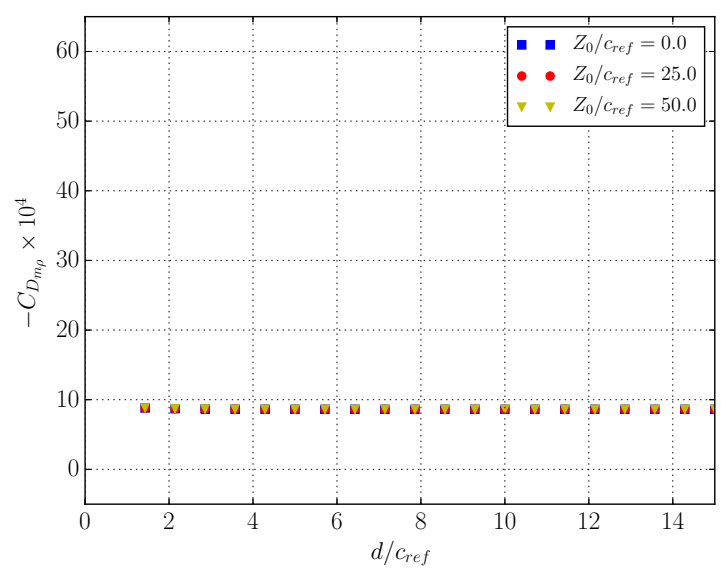

(d) $Z \rightarrow Z+Z_{0}$, new formula

Fig. $13 C_{D_{m_{\rho}}}$ evolutions for the wing of the NASA CRM, $M_{\infty}=0.724, R e=3 \times 10^{6}$ and $\alpha=1.15^{\circ}$ 


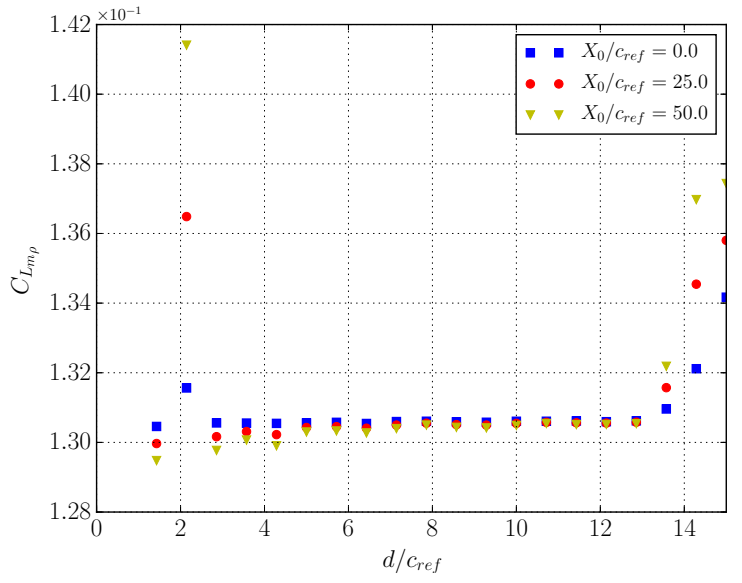

(a) $X \rightarrow X+X_{0}$, old formula

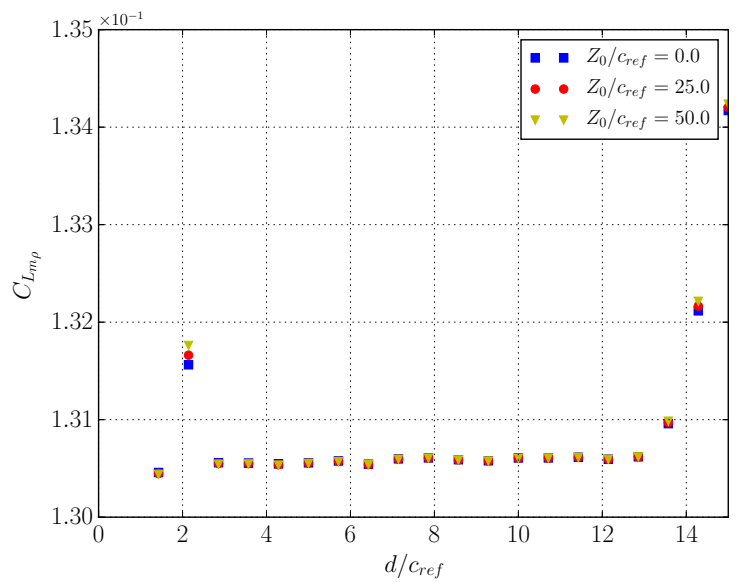

(c) $Z \rightarrow Z+Z_{0}$, old formula

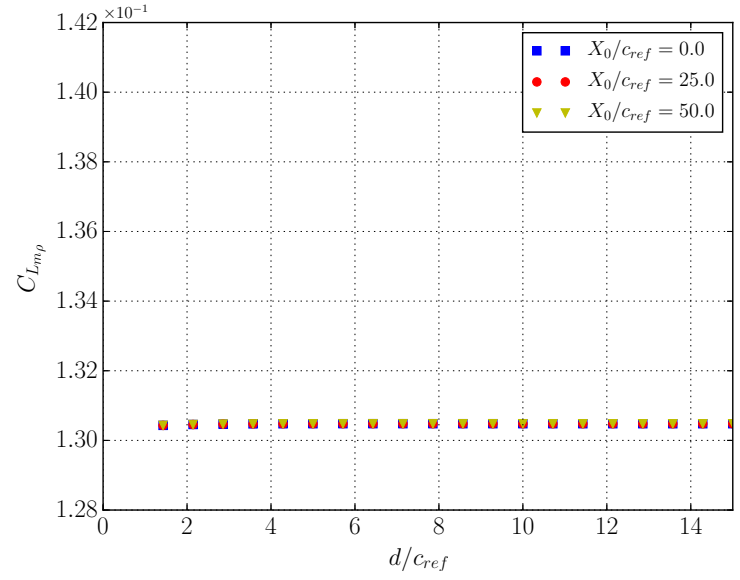

(b) $X \rightarrow X+X_{0}$, new formula

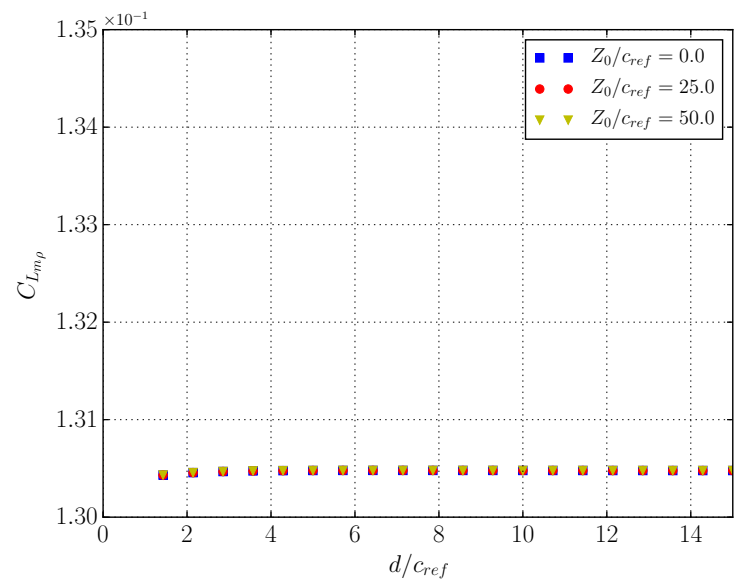

(d) $Z \rightarrow Z+Z_{0}$, new formula

Fig. $14 C_{L_{m_{\rho}}}$ evolutions for the wing of the NASA CRM, $M_{\infty}=0.724, \operatorname{Re}=3 \times 10^{6}$ and $\alpha=1.15^{\circ}$

\section{Conclusion}

In the present paper, the focus has been set on the dependence on the reference point of the Lamb vector based aerodynamic force breakdown. In the recent years, this formulation proved to be very promising since it defines the lift and lift-induced drag in compressible viscous flows. However, the sensitivity to the position of the reference point was still an open issue which questioned the robustness of the method and its potential use. The present study proposes a way to get rid of this dependence by considering the symmetries satisfied in the far field and extending it to the whole domain such that the additional terms appearing when proceeding to a shift in the reference point vanish. It consists in integrating the part of the physical vector fields which satisfy the symmetries everywhere in the flow. This systematic method may also be applied to surfaces that do not necessarily fulfill symmetries since the method presented above consists in applying a symmetry operation to the surface.

The numerical applications were carried out on two-dimensional and three-dimensional cases which allowed the present authors to verify the validity of the method. The results suggest that enforcing the symmetry properties everywhere in the control volume make the formulation invariant such that the reference point adopted for the computation of moments can be chosen arbitrarily. Therefore, the reference point invariant formulation guarantees more robustness for the Lamb vector based aerodynamic force breakdown.

However, there are still flaws to be addressed. Indeed, further research will be conducted on the sensitivity to the 
integration domain since the decomposition varies with the size of the control volume. Many former studies have focused on this point but none has found a way to undermine this feature. Furthermore, the computation of the wave drag is still an open issue.

\section{Appendix}

\section{A. Derivative Moment Transformations}

Let $\vec{f}$ be a differentiable vector field, $\Phi$ be a differentiable scalar field, $\Omega$ be a domain bounded by $\partial \Omega$ and $S$ be a surface bounded by $\partial S$. The derivative moment transformations state that in $n$-dimensional space with $n=2,3$ :

$$
\begin{gathered}
\int_{\Omega} \vec{f} \mathrm{~d} v=\frac{1}{n-1} \int_{\Omega} \vec{r} \times(\nabla \times \vec{f}) \mathrm{d} v-\frac{1}{n-1} \int_{\partial \Omega} \vec{r} \times(\vec{n} \times \vec{f}) \mathrm{d} S \\
\int_{S} \Phi \vec{n} \mathrm{~d} S=-\frac{1}{n-1} \int_{S} \vec{r} \times(\vec{n} \times \nabla \Phi) \mathrm{d} S+\frac{1}{n-1} \oint_{\partial S} \Phi \vec{r} \times \mathrm{d} \vec{r}
\end{gathered}
$$

Those identities are $\vec{r}_{0}$-independent: a shift $\vec{r} \rightarrow \vec{r}+\vec{r}_{0}$ gives birth to two additional terms that cancel each other according to Gauss theorem. However, the right-hand side integrals depend on the choice of $\vec{r}_{0}$.

\section{B. Far wake symmetry properties of the velocity vector derivatives}

Let $\mathcal{F}$ be a differentiable tensor and $\left(x_{1}, \ldots, x_{i}, \ldots, x_{n}\right)$ be a cartesian coordinate system. Then, the derivative of $\mathcal{F}$ with respect to $x_{i}$ writes:

$$
\frac{\partial \mathcal{F}}{\partial x_{i}}\left(x_{1}, \ldots, x_{i}, \ldots, x_{n}\right)=\lim _{\epsilon \rightarrow 0} \frac{\mathcal{F}\left(x_{1}, \ldots, x_{i}+\epsilon, \ldots x_{n}\right)-\mathcal{F}\left(x_{1}, \ldots, x_{i}, \ldots x_{n}\right)}{\epsilon}
$$

Therefore, the derivative of $\mathcal{F}$ with respect to $x_{i}$ assessed in $\left(x_{1}, \ldots,-x_{i}, \ldots, x_{n}\right)$ writes:

$$
\begin{aligned}
\frac{\partial \mathcal{F}}{\partial x_{i}}\left(x_{1}, \ldots,-x_{i}, \ldots, x_{n}\right) & =\lim _{\epsilon \rightarrow 0} \frac{\mathcal{F}\left(x_{1}, \ldots,-x_{i}+\epsilon, \ldots x_{n}\right)-\mathcal{F}\left(x_{1}, \ldots,-x_{i}, \ldots x_{n}\right)}{\epsilon} \\
& =\lim _{\epsilon \rightarrow 0} \frac{\mathcal{F}\left(x_{1}, \ldots,-\left(x_{i}-\epsilon\right), \ldots x_{n}\right)-\mathcal{F}\left(x_{1}, \ldots,-x_{i}, \ldots x_{n}\right)}{\epsilon}
\end{aligned}
$$

Starting from Eqs.(17a) and (17b) and using Eqs. (48) and (49), it is possible to derive the symmetry properties satisfied by the velocity vector derivatives:

$$
\left\{\begin{array} { l } 
{ \frac { \partial \vec { u } } { \partial x } ( x , - y , z ) = \vec { \vec { S } } _ { y } \cdot \frac { \partial \vec { u } } { \partial x } ( x , y , z ) } \\
{ \frac { \partial \vec { u } } { \partial y } ( x , - y , z ) = - \vec { \vec { S } } _ { y } \cdot \frac { \partial \vec { u } } { \partial y } ( x , y , z ) } \\
{ \frac { \partial \vec { u } } { \partial z } ( x , - y , z ) = \vec { \vec { S } } _ { y } \cdot \frac { \partial \vec { u } } { \partial z } ( x , y , z ) }
\end{array} \quad \text { and } \quad \left\{\begin{array}{l}
\frac{\partial \vec{u}}{\partial x}(x, y,-z)=\overrightarrow{\vec{S}}_{y} \cdot \frac{\partial \vec{u}}{\partial x}(x, y, z) \\
\frac{\partial \vec{u}}{\partial y}(x, y,-z)=\overrightarrow{\vec{S}}_{y} \cdot \frac{\partial \vec{u}}{\partial y}(x, y, z) \\
\frac{\partial \vec{u}}{\partial z}(x, y,-z)=-\overrightarrow{\vec{S}}_{y} \cdot \frac{\partial \vec{u}}{\partial z}(x, y, z)
\end{array}\right.\right.
$$

\section{Acknowledgments}

Part of this research was supported by a DGA scholarship.

\section{References}

[1] Paparone, L., and Tognaccini, R., "Computational Fluid Dynamics-Based Drag Prediction and Decomposition," AIAA Journal, Vol. 41, No. 9, 2003, pp. 1647-1657.

[2] Destarac, D., and Van Der Vooren, J., "Drag/thrust analysis of jet-propelled transonic transport aircraft; definition of physical drag components," Aerospace science and technology, Vol. 8, No. 6, 2004, pp. 545-556.

[3] Oswatitsch, K., Gas dynamics, Vol. 1, Academic Press, 1956.

[4] Wu, J., Liu, L., and Liu, T., "Fundamental theories of aerodynamic force in viscous and compressible complex flows," Progress in Aerospace Sciences, 2018. 
[5] Prandtl, L., “Theory of lifting surfaces," NACA TN 10, 1920.

[6] Saffman, P. G., Vortex dynamics, Cambridge university press, 1992.

[7] Wu, J.-Z., Ma, H.-Y., and Zhou, M.-D., Vorticity and vortex dynamics, Springer Science \& Business Media, 2007.

[8] Wu, J.-Z., Lu, X.-Y., and Zhuang, L.-X., "Integral force acting on a body due to local flow structures," Journal of Fluid Mechanics, Vol. 576, 2007, pp. 265-286.

[9] Liu, L., Wu, J., Shi, Y., and Zhu, J., “A dynamic counterpart of Lamb vector in viscous compressible aerodynamics,” Fluid Dynamics Research, Vol. 46, No. 6, 2014, p. 061417.

[10] Mele, B., and Tognaccini, R., "Aerodynamic force by Lamb vector integrals in compressible flow," Physics of Fluids, Vol. 26, No. 5, 2014, p. 056104.

[11] Marongiu, C., Tognaccini, R., and Ueno, M., "Lift and lift-induced drag computation by Lamb vector integration," AIAA journal, Vol. 51, No. 6, 2013, pp. 1420-1430.

[12] Yang, Y., Zhang, R., An, Y., and Wu, J., "Steady vortex force theory and slender-wing flow diagnosis," Acta Mechanica Sinica, Vol. 23, No. 6, 2007, pp. 609-619.

[13] Mele, B., Ostieri, M., and Tognaccini, R., "Aircraft lift and drag decomposition in transonic flows," Journal of Aircraft, Vol. 54, No. 5, 2017, pp. 1933-1944.

[14] Ostieri, M., and Tognaccini, R., "On a recently proposed vorticity-based definition of wave drag," Journal of Aircraft, Vol. 55, No. 6, 2018, pp. 2521-2523.

[15] Mele, B., Ostieri, M., and Tognaccini, R., "Vorticity based breakdown of the aerodynamic force in three-dimensional compressible flows," AIAA Journal, , No. null, 2016, pp. 1198-1208.

[16] Kang, L., Russo, L., Tognaccini, R., Wu, J., and Su, W., “Aerodynamic force breakdown based on vortex force theory,” AIAA Scitech 2019 Forum, 2019, p. 2122.

[17] Devenport, W. J., Rife, M. C., Liapis, S. I., and Follin, G. J., “The structure and development of a wing-tip vortex,” Journal of Fluid Mechanics, Vol. 312, 1996, pp. 67-106.

[18] Batchelor, G., “Axial flow in trailing line vortices," Journal of Fluid Mechanics, Vol. 20, No. 04, 1964, pp. 645-658.

[19] Fabre, D., "Instabilité et instationnarités dans les tourbillons: Application aux sillages d'avions," Ph.D. thesis, Université Paris 6, 2002.

[20] Misaka, T., Holzäpfel, F., and Gerz, T., "Large-eddy simulation of aircraft wake evolution from roll-up until vortex decay," AIAA Journal, Vol. 53, No. 9, 2015, pp. 2646-2670.

[21] Liu, L., Kang, L., and Wu, J., “Zonal structure of unbounded external-flow and aerodynamics," Fluid Dynamics Research, Vol. 49, No. 4, 2017, p. 045508.

[22] Liu, L.-Q., Unified Theoretical Foundations of Lift and Drag in Viscous and Compressible External Flows, Springer, 2017.

[23] Sipp, D., Jacquin, L., and Cosssu, C., "Self-adaptation and viscous selection in concentrated two-dimensional vortex dipoles," Physics of Fluids, Vol. 12, No. 2, 2000, pp. 245-248.

[24] Wu, J.-Z., Ma, H.-Y., and Zhou, M.-D., Vortical flows, Vol. 28, Springer, 2015. 